\title{
Phosphorylation of Synaptojanin Differentially Regulates Endocytosis of Functionally Distinct Synaptic Vesicle Pools
}

\author{
이 Junhua Geng, ${ }^{1 \star}$ Liping Wang, ${ }^{1,2 *}$ Joo Yeun Lee, ${ }^{1,4}$ CChun-Kan Chen, ${ }^{1}$ and Karen T. Chang ${ }^{1,3,4}$ \\ 'Zilkha Neurogenetic Institute, ${ }^{2}$ Department of Biochemistry and Molecular Biology, and ${ }^{3}$ Department of Cell and Neurobiology, Keck School of Medicine, \\ University of Southern California, Los Angeles, California 90089, and ${ }^{4}$ Neuroscience Graduate Program, University of Southern California, Los Angeles, \\ California 90089
}

The rapid replenishment of synaptic vesicles through endocytosis is crucial for sustaining synaptic transmission during intense neuronal activity. Synaptojanin (Synj), a phosphoinositide phosphatase, is known to play an important role in vesicle recycling by promoting the uncoating of clathrin following synaptic vesicle uptake. Synj has been shown to be a substrate of the minibrain (Mnb) kinase, a fly homolog of the dual-specificity tyrosine phosphorylation-regulated kinase 1A (DYRK1A); however, the functional impacts of Synj phosphorylation by Mnb are not well understood. Here we identify that Mnb phosphorylates Synj at S1029 in Drosophila. We find that phosphorylation of Synj at S1029 enhances Synj phosphatase activity, alters interaction between Synj and endophilin, and promotes efficient endocytosis of the active cycling vesicle pool (also referred to as exo-endo cycling pool) at the expense of reserve pool vesicle endocytosis. Dephosphorylated Synj, on the other hand, is deficient in the endocytosis of the active recycling pool vesicles but maintains reserve pool vesicle endocytosis to restore total vesicle pool size and sustain synaptic transmission. Together, our findings reveal a novel role for Synj in modulating reserve pool vesicle endocytosis and further indicate that dynamic phosphorylation and dephosphorylation of Synj differentially maintain endocytosis of distinct functional synaptic vesicle pools.

Key words: endocytosis; minibrain; phosphorylation; synaptojanin

\section{Significance Statement}

Synaptic vesicle endocytosis sustains communication between neurons during a wide range of neuronal activities by recycling used vesicle membrane and protein components. Here we identify that Synaptojanin, a protein with a known role in synaptic vesicle endocytosis, is phosphorylated at S1029 in vivo by the Minibrain kinase. We further demonstrate that the phosphorylation status of Synaptojanin at S1029 differentially regulates its participation in the recycling of distinct synaptic vesicle pools. Our results reveal a new role for Synaptojanin in maintaining synaptic vesicle pool size and in reserve vesicle endocytosis. As Synaptojanin and Minibrain perturbations are associated with various neurological disorders, such as Parkinson's, autism, and Down syndrome, understanding mechanisms modulating Synaptojanin function provides valuable insights into processes affecting neuronal communication.

\section{Introduction}

During synaptic activity, the rapid recycling of synaptic vesicle through endocytosis enable neurons to maintain stable synaptic

\footnotetext{
Received May 4, 2016; revised July 11, 2016; accepted July 14, 2016.

Author contributions: K.T.C. designed research; J.G., L.W., J.Y.L., and C.-K.C. performed research; J.G., L.W., J.Y.L., C.-K.C., and K.T.C. analyzed data; J.G., L.W., and K.T.C. wrote the paper.

This work was supported by National Institutes of Health Grant NS080946 and Alzheimer's Association and Global Down Syndrome Foundation to K.T.C. We thank Martin Heisenberg (University of Wuzburg, Wuzburg, Germany) for the $m n b^{7}$ stock; Hugo Bellen (Baylor University, Waco, Texas) for generous sharing of synj ${ }^{7}$ and synj ${ }^{2}$ flies, endophilin antibody, and synaptojanin antibody, which we renamed here to p-Synj for clarification; Syed Qadri for performing preliminary experiments; and the Developmental Studies Hybridoma Bank (lowa City, lowa) for antibodies.

The authors declare no competing financial interests.

*J.G. and L.W. contributed equally to this study.

Correspondence should be addressed to Dr. Karen T. Chang, Zilkha Neurogenetic Institute, Keck School of Medicine, University of Southern California, Los Angeles, CA 90089. E-mail: changkt@usc.edu.
}

transmission across a wide range of stimulation frequencies without exhausting their supply of synaptic vesicles (Saheki and De Camilli, 2012; Soykan et al., 2016). There are at least two distinct synaptic vesicle pools at the Drosophila neuromuscular junction $(\mathrm{NMJ})$ : the active recycling pool also known as the exo/endo recycling pool (ECP) and the reserve vesicle pool (RP) (Kuromi and Kidokoro, 1998, 2000, 2002; Delgado et al., 2000; Rizzoli and Betz, 2005). The ECP vesicles are retrieved rapidly during synaptic activity and include the readily releasable pool and the recycling vesicles, both of which contribute to neurotransmitter

C.-K. Chen's present address: Division of Biology and Biological Engineering, California Institute of Technology, Pasadena, CA 91125.

DOI:10.1523/JNEUROSCI.1470-16.2016

Copyright $\odot 2016$ the authors $\quad 0270-6474 / 16 / 368882-13 \$ 15.00 / 0$ 
release at low stimulation frequency or high $\mathrm{K}^{+}$depolarization (Kuromi and Kidokoro, 2005; Verstreken et al., 2005). The RP is recruited only during high-frequency nerve stimulation and is thought to refill slowly after cessation of synaptic stimulation (Kuromi and Kidokoro, 2002; Verstreken et al., 2005; Akbergenova and Bykhovskaia, 2009). Both the ECP and RP are required for normal synaptic transmission (Kuromi and Kidokoro, 1998, 2002; Verstreken et al., 2005). Although a vast array of proteins, including kinases and phosphatases, have been identified to coordinate synaptic vesicle retrieval and recycling through a series of precisely controlled events, whether they differentially affect the ECP, RP, or both, are less understood.

Synaptojanin (Synj) is a phosphoinositide phosphatase known to play an important role in synaptic vesicle recycling (McPherson et al., 1996; Cremona et al., 1999; Harris et al., 2000; Verstreken et al., 2003; Mani et al., 2007). Mutations in Synj cause a significant depletion of synaptic vesicles and an accumulation of densely coated synaptic vesicles in both vertebrates and invertebrates, suggesting Synj is crucial for the uncoating of clathrin during clathrin-mediated endocytosis (Cremona et al., 1999; Haffner et al., 2000; Harris et al., 2000; Verstreken et al., 2003). Synj has two phosphoinositol phosphatase domains that regulate the levels of phosphoinositide pools, as well as a proline rich domain (PRD) that interacts with endocytic proteins containing a Src Homology 3 (SH3) domain, such as endophilin (McPherson et al., 1996; Ringstad et al., 1997; Schuske et al., 2003; Verstreken et al., 2003). Aside from coordinating protein interactions, the PRD of Synj is a site of post-translational modulation of Synj activity. Phosphorylation of Synj by Cdk5 has been shown to inhibit Synj phosphatase activity (Lee et al., 2004). We have also demonstrated that phosphorylation of Synj by the Mnb kinase (also known as Dyrk1A), enhances Synj activity and is required for reliable synaptic vesicle recycling (Chen et al., 2014). However, the site on Synj phosphorylated by Mnb has not been identified, and the precise functional impact of Mnb-dependent phosphorylation of Synj in regulating synaptic vesicle recycling remains unclear. Interestingly, both Mnb and Synj are overexpressed in Down syndrome (Guimera et al., 1999; Arai et al., 2002; Dowjat et al., 2007), and Synj and Mnb mutations have been linked to Parkinson's disease and Autism (Iossifov et al., 2012; O’Roak et al., 2012; Krebs et al., 2013), respectively. An understanding of Mnb and Synj functional interactions may thus shed light on mechanisms underlying these neurological disorders.

In the present study, we demonstrate that the Mnb kinase phosphorylates Synj at S1029 in vivo. Phosphorylation of S1029 by Mnb decreases Synj-Endophilin interactions and enhances Synj phosphatase activity. FM1-43 labeling experiments revealed that phosphorylation of Synj at S1029 is necessary for ECP endocytosis but surprisingly is not required to maintain stable synaptic transmission during high-frequency stimulation. We find nonphosphorylated Synj can still maintain RP endocytosis to compensate for defects in ECP endocytosis, whereas phosphorylation of Synj at S1029 promotes endocytosis of the ECP at the expense of RP endocytosis. Together, these data reveal that Synj participates in the recycling of both the ECP and RP vesicles and that the phosphorylation status of Synj differentially regulates endocytosis of distinct functional vesicle pools at the Drosophila NMJ.

\section{Materials and Methods}

Fly stocks and antibody generation. Flies were cultured at $25^{\circ} \mathrm{C}$ on standard cornmeal, yeast, sugar, and agar medium under a $12 \mathrm{~h} \mathrm{light} \mathrm{and} 12 \mathrm{~h}$ dark cycle. The following fly lines were used: $s y n j^{1}$ and $s y n j^{2}$ (From Dr. Hugo Bellen), $m n b^{1}$ (from Dr. Martin Heisenberg), and UAS-PLCס1-
PH-GFP (Bloomington stock center \#39693). UAS-synj ${ }^{w t}$ was generated as described by Chen et al. (2014) but is a different transgenic line with insertion in the third chromosome. Synj ${ }^{\text {S1029A }}$ and Synj ${ }^{\text {S1029E }}$ transgene constructs were generated using site-directed mutagenesis and were cloned into the pINDY6 vector, which contains a HA tag. Transgenic flies were generated via standard P-element transformation method (Montell et al., 1985). To drive neuronal expression, n-synaptobrevin-Gal4 (nSynb-Gal4) (Pauli et al., 2008) was used (gift from Julie Simpson). $n S y n b-G a l 4, U A S-P L C \delta 1-P H-G F P$ was generated by recombination. All other stocks and standard balancers were obtained from Bloomington Stock Center. Flies of both sexes were used, except for experiments shown in Figure $2 F, G$, in which only male flies were used and compared ( $m n b$ located on the X chromosome). Polyclonal phospho-Synj ${ }^{\text {S1029 }}$ antibody was generated by immunizing rabbits with synthetic peptide phosphorylated at serine corresponding to Synj ${ }^{\mathrm{S} 1029}:{ }^{1027} \mathrm{PMSP}$ KNSPRHLP ${ }^{1038}$ (PrimmBiotech). Phospho-specific antibody was purified by nonphospho-peptide depletion column, followed by affinity purification using phospho-peptide affinity column. Specificity against phosphorylated antigen was confirmed by dot assays using phosphorylated antigen and nonphosphorylated antigen.

Mass spectrometry. His-Synj was purified and treated with alkaline phosphatase with or without Mnb as described previously and below (Chen et al., 2014). Mass spectrophotometry was performed as described previously (Chen et al., 2014). Samples were analyzed using an LC/MS system consisting of an Eksigent NanoLC Ultra 2D and Thermo Fisher Scientific LTQ Orbitrap XL. Proteome Discoverer 1.4 (Thermo Fisher Scientific) was used for protein identification using Sequest algorithms. The following criteria were followed. For MS/MS spectra, variable modifications were selected to include N,Q deamination, M oxidation, and C carbamidomethylation with a maximum of four modifications. Searches were conducted against Uniprot or in-house customer database. Oxidized methionines and phosphorylation of tyrosine, serine, and threonine were set as variable modifications. For the proteolytic enzyme, up to two missed cleavages were allowed. Up to two missed cleavages were allowed for protease digestion and peptide had to be fully tryptic. MS1 tolerance was $10 \mathrm{ppm}$ and MS2 tolerance was set at 0.8 Da. Peptides reported via search engine were accepted only if they met the false discovery rate of $1 \%$. There is no fixed cutoff score threshold, but instead spectra are accepted until the $1 \%$ false discovery rate is reached. Only peptides with a minimum of six amino acid lengths were considered for identification. We also validated the identifications by manual inspection of the mass spectra.

Synj PRD construction. Synj PRD constructs were cloned into PET15b vector (Novagen) between NdeI and XhoI sites. PRD-Full contains the full PRD of Synj, which covers amino acids 977-1218 of Synj. PRD-1 contains amino acids 977-1042, PRD-2 covers amino acids 1043-1091, PRD-3 contains amino acids 1092-1139, PRD-4 contains 1140-1218 of synj. BL21(DE3) competent Escherichia coli. strain containing the expression plasmids were grown at $37^{\circ} \mathrm{C}$ until $\mathrm{A} 600$ of the culture reached $0.6-0.8$. Expression of the proteins was induced by the addition of isopropyl $\beta$-D-thiogalactopyranoside to a final concentration of $1.0 \mathrm{~mm}$. After growth at $30^{\circ} \mathrm{C}$ for $4 \mathrm{~h}$, cells were harvested and stored at $-80^{\circ} \mathrm{C}$ until purification. Ni-NTA Purification System (Invitrogen) was used to purify His-PRD proteins.

Immunochemistry. Third-instar larvae were dissected in $\mathrm{Ca}^{2+}$ free dissection buffer: $128 \mathrm{~mm} \mathrm{NaCl}, 2 \mathrm{~mm} \mathrm{KCl}, 4.1 \mathrm{~mm} \mathrm{MgCl}_{2}$, $35.5 \mathrm{~mm}$ sucrose, 5 mM HEPES, 1 mм EGTA. Motor nerves were cut and dissected preparations were fixed in $4 \%$ PFA solution for $20 \mathrm{~min}$ at room temperature. Fixed samples were then washed with $0.1 \%$ Triton X-100 in PBS (PBST) and blocked with 5\% normal goat serum in PBST. Dissected preps were incubated with primary antibodies diluted in blocking solution overnight at $4^{\circ} \mathrm{C}$. Antibodies were diluted as following: rabbit anti-Synj-1, 1:200; rabbit anti-p-Synj, 1:2000; mouse anti-Dlg (4F3), 1:500 (developmental Studies Hybridoma Bank); Cy3-conjugated anti-HRP, 1:100 (Jackson ImmunoResearch Laboratories). Secondary antibodies used were Alexa-488 or 405 conjugated, 1:250 (Invitrogen).

Image quantification. Images of synaptic terminals from NMJ 6/7 in segment A2 and A3 were captured by using a Zeiss LSM5 confocal microscope with a $\times 631.6$ numerical aperture oil-immersion objective. 
Staining intensities were measured by normalizing the fluorescence intensity to bouton area outlined by HRP using ImageJ. Exposure time was kept consistent for all genotypes per experiment within same day while comparing intensity across genotypes. All values were normalized to control done within each experimental set.

Immunoprecipitation and protein interaction. Fly heads (200) were homogenized in lysis buffer (10 mm HEPES, $100 \mathrm{~mm} \mathrm{NaCl}, 10 \mathrm{~mm}$ EDTA, $1 \% \mathrm{NP}-40,1 \mathrm{~mm} \mathrm{Na} \mathrm{VO}_{4}, 50 \mathrm{~mm} \mathrm{NaF}, 250 \mathrm{~nm}$ cyclosporin A, and protease inhibitor) and centrifuged to remove debris. Anti-HA-Agarose (Sigma) $(20 \mu \mathrm{l})$ were added to the extracts and rotated at $4^{\circ} \mathrm{C}$ overnight. After three washes in Lysis buffer, the immunocomplexes were eluted with SDS sample buffer, and all of the eluates were used for Western blotting. Primary antibodies were diluted in blocking solution as following: rabbit anti-HA, 1:200; guinea pig anti-Endo (GP60), 1:5000. All values were normalized to total Synj within the same experimental set. Intensity of each band was quantified using ImageJ.

In vitro phosphorylation of Synj. Synj were immunoprecipitated using HA agarose beads from fly heads as described above, and then dephosphorylated with alkaline phosphatase in CutSmart Buffer (New England BioLabs) at $37^{\circ} \mathrm{C}$ for $1 \mathrm{~h}$. Dephosphorylated Synj were then washed with kinase buffer (20 mm HEPES, pH 7.4, $10 \mathrm{~mm} \mathrm{MgCl}_{2}, 1 \mathrm{~mm}$ DTT and $2 \mathrm{~mm}$ $\mathrm{Na}_{3} \mathrm{VO}_{4}$ ) four times to remove CIP. For Mnb rephosphorylation, samples were subsequently treated with purified Mnb $(0.5 \mu \mathrm{g})$ in kinase buffer plus $50 \mu \mathrm{M} \mathrm{ATP}$ at $37^{\circ} \mathrm{C}$ for $1 \mathrm{~h}$ and then washed with PBS for four times to remove Mnb. Synj were eluted with SDS sample buffer and used for Western blotting. p-Ser/Thr-Pro antibody was purchased from EMD Millipore and used at 1:1500.

Phosphatidylinositol $5^{\prime}$-phosphatase activity in vitro. Determination of Synj phosphotidylinositol 5' -phosphatase activity was previously described (Chen et al., 2014). Adult fly heads were homogenized in Lysis buffer and Synj immunoprecipitated using HA agarose beads. Purified Synj was incubated with labeled PI(4,5)P2 (GloPIPs BODIPY FL$\mathrm{PI}(4,5) \mathrm{P} 2$, Echelon) in inositol phosphatase activity assay buffer $(30 \mathrm{~mm}$ HEPES, pH 7.4, $100 \mathrm{~mm} \mathrm{KCl,} 1 \mathrm{~mm}$ EGTA and $2 \mathrm{~mm} \mathrm{MgCl}_{2}$ ) at room temperature for 5-10 min. Lipid products were separated by TLC and visualized under ultraviolet radiation. Synj was eluted with SDS sample buffer, and Synj level was determined by Western blotting. Phosphotidylinositol 5-phosphatase activity was normalized to the level of Synj.

In vivo phosphatidylinositol $5^{\prime}$-phosphatase activity determination. Third instar larvae were dissected in $\mathrm{Ca}^{2+}$ free dissection buffer with motor nerves intact. Dissected preps were fixed for $20 \mathrm{~min}$ in 4\% PFA, washed with PBS, and then incubated with HRP-Cy3 for $15 \mathrm{~min}$. Images were taken right away using a Zeiss LSM5 confocal microscope. PLC $\delta$-PH-eGFP fluorescence intensity was analyzed by outlining the bouton area using HRP in ImageJ. All values were normalized to control done within each experimental set.

Viability assay. Fly eggs were collected from apple juice plates and then transferred to standard fly medium. Viable third instar larvae with the right genotype were picked and counted between day 3 and day 7 , and was then used to determine percentage viability by normalizing to the number of eggs predicted to have the right genotype. For each genotype, a minimum of 1440 eggs was used. All experiments were conducted at $25^{\circ} \mathrm{C}$ and $75 \%$ relative humidity, in $12 \mathrm{~h}$ of light per day.

FM1-43 dye labeling. Third-instar larvae were dissected in modified HL-3 $\mathrm{Ca}^{2+}$ free solution: $70 \mathrm{~mm} \mathrm{NaCl}, 5 \mathrm{~mm} \mathrm{KCl}, 10 \mathrm{~mm} \mathrm{MgCl} 2,10 \mathrm{~mm}$ $\mathrm{NaHCO}_{3}, 5$ mm Trehalose, $115 \mathrm{~mm}$ sucrose, 5 mm HEPES, 2.5 mm EGTA. For high potassium stimulation, dissected samples were incubated with 4 $\mu \mathrm{M}$ FM1-43 dye (Invitrogen) in modified HL-3 solution containing 60 $\mathrm{mm} \mathrm{KCl}$ and $2 \mathrm{~mm} \mathrm{CaCl}_{2}$ for $5 \mathrm{~min}$, then washed with modified HL-3 $\mathrm{Ca}^{2+}$ free solution. For reserve vesicle pool loading, dissected samples were bathed in $4 \mu \mathrm{M}$ FM1-43 dye (Invitrogen) in modified HL-3 solution containing $2 \mathrm{mM} \mathrm{CaCl}_{2}$, and segmental motor nerves were stimulated using a suction electrode at $10 \mathrm{~Hz}$ for $10 \mathrm{~min}$ plus an additional $5 \mathrm{~min}$ wait without stimulation. Samples were washed with modified HL-3 $\mathrm{Ca}^{2+}$ free solution. For unloading experiment in both high potassium and electrical stimulation, larvae were incubated with modified HL-3 solution containing $60 \mathrm{~mm} \mathrm{KCl}$ and $2 \mathrm{mM} \mathrm{CaCl}_{2}$ for $1 \mathrm{~min}$ and $5 \mathrm{~min}$, respectively. To directly monitor RP vesicle loading, larval prep was immediately incubated with FM1-43 dye in $\mathrm{Ca}^{2+}$ free HL-3 for 5 min at the cessation of $10 \mathrm{~Hz}$ electrical stimulation. Images were taken using Zeiss LSM 5 confocal microscope with a $40 \times$ water-dipping objective. Fluorescence intensities were calculated using Image and normalized to the average loading fluorescence intensity in controls within the same experimental set. The ratio of unloaded/loaded was calculated by subtracting the fluorescence intensity remained after unloading from the loading intensity, then divided by loaded fluorescence intensity: $\left(\mathrm{F}_{\text {loaded }}-\mathrm{F}_{\text {unloaded }}\right) / \mathrm{F}_{\text {loaded }}$. The ratio of reserve vesicle pool was calculated by fluorescence intensity remaining after unloading divided by loaded fluorescence intensity: $\mathrm{F}_{\text {unloaded }} / \mathrm{F}_{\text {loaded }}$.

Electrophysiology. Third-instar larvae were dissected in modified HL-3 $\mathrm{Ca}^{2+}$ free solution. Dissected larvae were bathed in modified HL-3 solution containing 0.4 or $2 \mathrm{mM} \mathrm{Ca}^{2+}$ as indicated. Current-clamp recordings were performed on muscles 6 in abdominal segments $\mathrm{A} 2$ or $\mathrm{A} 3$, and severed ventral nerves were stimulated with suction electrodes at $0.3 \mathrm{~ms}$ stimulus duration. The recording electrode with resistance between 20 and $40 \mathrm{M} \Omega$ was filled with $3 \mathrm{M} \mathrm{KCl}$. Recordings were discarded if $V_{\mathrm{m}}$ changed by $>10 \%$ and also were rejected in which the stimulated nerve did not function fully throughout the recording, which was determined by abrupt drops in EPSP amplitude. Data were acquired using an Axopatch 200B amplifier, digitized using a Digidata 1440A, and controlled using pClamp 10.3 software (Molecular Devices). Electrophysiological sweeps were sampled at a rate of $10 \mathrm{kHz}$ and filtered at $400 \mathrm{~Hz}$. Data were analyzed using MiniAnalysis (Synaptosoft), SigmaPlot (Systat Software), and Microsoft Excel. Nonlinear summation was used to correct the average EPSP amplitude. Bafilomycin A1 (Adipogen) and 1-(5iodonaph-thalene-1 sulfonly1)-1H-hexahydro-1,4-diazepine hydrochloride (ML-7) (Sigma) were used at $2 \mu \mathrm{M}$ and $1 \mu \mathrm{M}$, respectively. For drug treatment, dissected preps were incubated with drug containing modified HL-3 solution ( $2 \mathrm{~mm} \mathrm{Ca}^{2+}$ ) for $30 \mathrm{~min}$ before recording. Vehicle control contains the same concentration of DMSO $(0.5 \%)$ as solution containing the respective drug.

Statistics. For paired samples, Student's $t$ test was used. For multiple samples, ANOVA test followed by Bonferroni post hoc test was used to determine statistical significance.

\section{Results}

\section{Minibrain kinase phosphorylates Synaptojanin at S1029 in vitro and in vivo}

Mnb is a proline-directed kinase previously shown to phosphorylate Synj and regulate synaptic vesicle endocytosis (Himpel et al., 2000; Adayev et al., 2006; Chen et al., 2014). To further delineate the effects of Synj phosphorylation by Mnb, we set out to identify the site(s) on Synj phosphorylated by Mnb. To this end, we purified HA-tagged Synj (Synj-HA) from bacteria, treated it with alkaline phosphatase to remove the existing phosphorylation, and then incubated Synj with or without purified Mnb kinase. LC-MS/MS was then performed to identify phosphorylation of Synj by Mnb kinase, which yielded 66\% coverage in Synj protein sequence. Serine 1029 (S1029) was the only Ser/Thr-Pro site differentially phosphorylated by Mnb with $>75 \%$ probability (Fig. $1 A)$. Treatment of purified Synj with alkaline phosphatase is necessary because it was shown that the majority of Synj purified from bacteria is phosphorylated (Chen et al., 2014). Figure $1 \mathrm{~A}$ further highlights that, although the PRD of Synj is not well conserved between fly and human, this potential Ser-Pro phosphorylation site is conserved.

To further confirm that Mnb kinase can indeed phosphorylate Synj at S1029, we generated transgenic flies with HA-tagged Synj phospho-null mutation $\left(\right.$ synj $^{\text {S1029A }}$ ) and HA-tagged Synj phospho-mimetic mutation $\left(s y n j^{S 1029 E}\right)$. First, we took an in vitro approach to test whether Mnb kinase does indeed phosphorylate Synj at S1029. Using an antibody generated against phosphory-

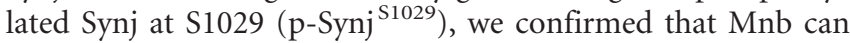
phosphorylate HA-tagged wild-type Synj (Synj ${ }^{\text {wt }}$ ) immunoprecipitated from fly heads (Fig. 1B). However, Mnb failed to phos- 
A
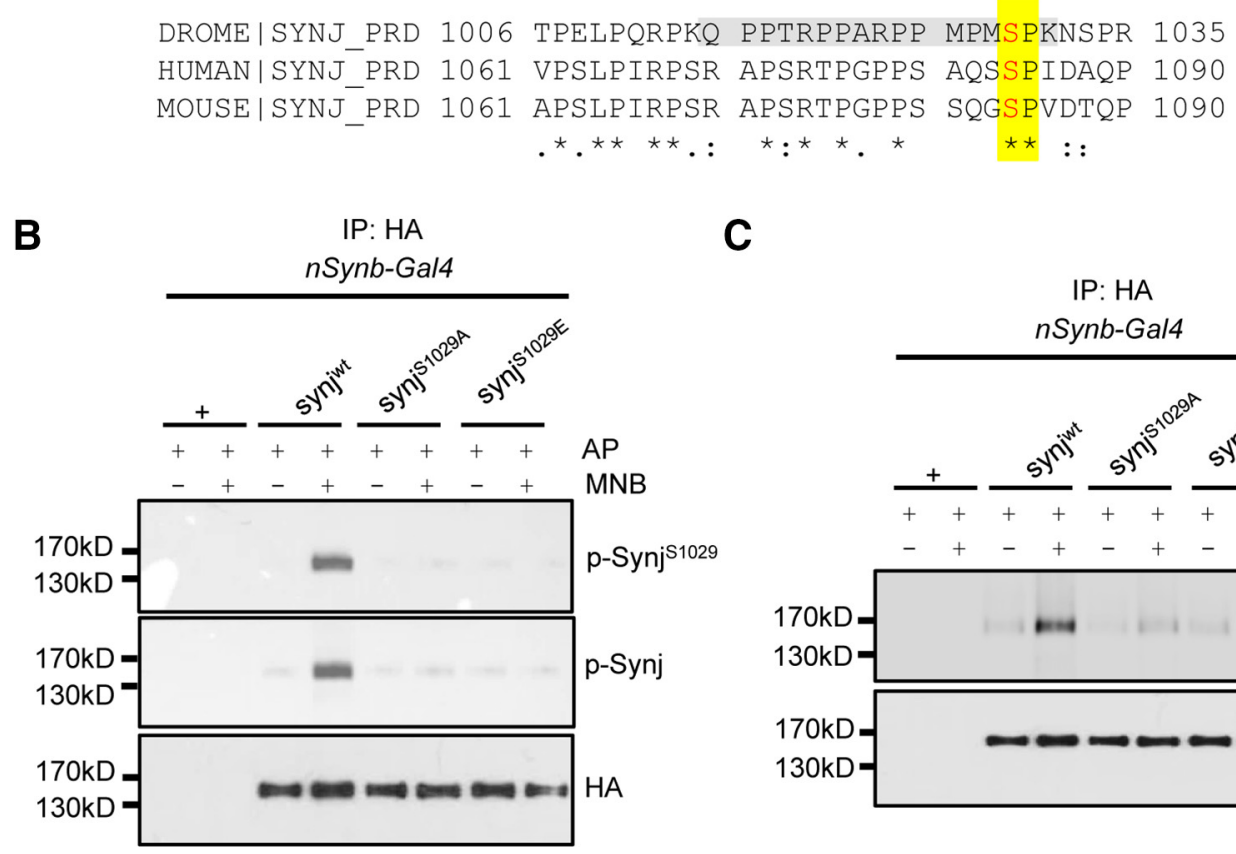

C

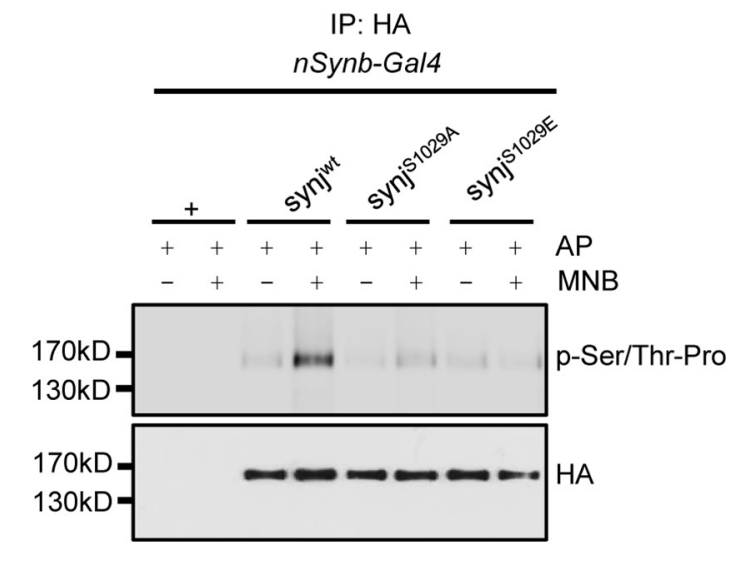

Figure 1. Mnb phosphorylates Synaptojanin at S1029 in vitro. A, Protein sequence alignment of partial Drosophila Synj-1 PRD domain with mouse and human Synj-1 PRD domain (Clustal Omega). Phosphorylation of Ser 1029 by Mnb precedes a Proline residue, consistent with Mnb being a proline-directed kinase (highlighted in yellow). Gray shaded region represents the peptide fragment identified from MS. B, C, Validation that Mnb phosphorylates Synj at Ser1029 using the indicated antibodies. HA-tagged Synj ${ }^{\text {wt }}$, Synj ${ }^{\text {S1029A }}$, and Synj ${ }^{\text {S1029E }}$ were expressed in flies, immunoprecipitated using HA-agarose beads, and incubated with or without purified Mnb kinase following alkaline phosphatase (AP) treatment. Representative blots are shown, and results were confirmed in at least 3 independent experiments. Mutation at S1029 abolished the increased phosphorylation by Mnb.

phorylate Synj $^{\text {S1029A }}$ or Synj ${ }^{\text {S1029E }}$, confirming that Mnb does indeed act on S1029 (Fig. 1B). Similar results were obtained using an antibody previously shown to be sensitive to Mnb-dependent phosphorylation of Synj (p-Synj; Fig. 1B), indicating p-Synj antibody is also sensitive to phosphorylation at Ser 1029 (Chen et al., 2014). To further determine whether Mnb acts on other sites in addition to Ser 1029, we performed the experiment using an antibody specific for phosphorylated Ser/Thr-Pro. Figure $1 C$ shows that incubation of Mnb with Synj increased the p-Ser/Thr-Pro signal detected for Synj ${ }^{\text {wt }}$, but not for Synj ${ }^{\text {S1029A }}$ or Synj ${ }^{\mathrm{S} 1029 \mathrm{E}}$. Together, these results demonstrate that Mnb phosphorylates Synj at S1029 and does not act on other Ser/ThrPro sites with high efficiency.

To investigate whether phosphorylation of Synj at S1029 indeed occurs in vivo, we first checked for the presence of p-Synj ${ }^{\text {S1029 }}$ signal in wild-type fly head protein extracts with and without alkaline phosphatase treatment. Figure $2 \mathrm{~A}$ shows that alkaline phosphatase treatment abolished the signal detected by p-SynjS1029, confirming that Synj is normally phosphorylated at S1029 in vivo. We also performed immunostaining at the NMJ using phospho-Synj antibodies. Unfortunately, our newly generated p-Synj ${ }^{\text {S1029 }}$ antibody did not work for immunostaining; we therefore used the p-Synj antibody described previously (Chen et al., 2014). We found that expression of Synj constructs led to an increase in the levels of total Synj and phospho-Synj staining compared with control (Fig. 2B,C). After normalizing the phospho-Synj signal to total Synj signal, we find that only Synj ${ }^{\text {wt }}$ expression showed a significant increase in relative phospho-Synj level, whereas Synj ${ }^{\text {S1029A }}$ and Synj ${ }^{\text {S1029E }}$ both did not (Fig. $2 C$ ). The fact that we observed an increase in phospho-Synj signal when S1029 is mutated suggests that this antibody may recognize another phosphorylation site other than S1029. We thus performed epitope mapping of the phospho-Synj antibody using truncated Synj PRD regions purified from bacteria (Fig. $2 D)$. Figure $2 E$ confirms that phospho-Synj antibody indeed recognizes and is sensitive to phosphorylation of two different regions of Synj: PRD-1, which covers S1029, as well as PRD-4. Because we did not detect differential phosphorylation of Synj by Mnb at another site when we mutated S1029 (Fig. 1), phosphorylation of PRD-4 region is independent of Mnb. Furthermore, these results suggest that the relative phospho-Synj to total Synj level better reflects the extent of Synj phosphorylation at $\mathrm{S} 1029$.

To further confirm that phosphorylation of Synj at S1029 is dependent on Mnb in vivo, we examined phospho-Synj signal in $m n b^{1}$ mutant background. Whereas $m n b$ null mutants are lethal, $m n b^{l}$ mutant harbors a point mutation that impairs kinase function (Tejedor et al., 1995; Chen et al., 2014; Ori-McKenney et al., 2016), leading to an overall decrease in phospho-Synj signal (Fig. 2 F, G). Indeed, expression of Synj ${ }^{\text {S1029A }}$ and Synj ${ }^{\text {S1029E }}$ constructs in $m n b^{1}$ mutant background resulted in the same reduced phospho-Synj to total Synj ratio as observed for $m n b^{1}$ mutant. Although expression of Synj ${ }^{\mathrm{wt}}$ in $m n b^{1}$ mutant background did not decrease the ratio of phospho-Synj to total Synj to that of $m n b^{l}$ level, it was nevertheless lower than expression in wild-type background (Fig. 2B). This result confirms that Mnb can phosphorylate Synj at S1029 in vivo. The subtle increase in phosphoSynj to total synj signal is likely caused by an increase in the amount of available substrate, Synj, because $m n b^{1}$ impairs rather than completely abolishes kinase activity (Tejedor et al., 1995; Chen et al., 2014). Together, our results indicate that Mnb can phosphorylate Synj at S1029, and that phosphorylation of Synj at S1029 occurs in vivo. 
A

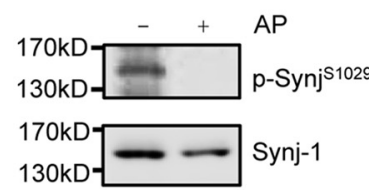

D PRD-1 977 VDSEIELCTT NTIPLCANPV EHAQLLQAIT PELPQRPKQP PRD-2 1017 PTRPPARPPM PMSPKNSPRH LPHVGVISIV PKPAKPPMPP 1057 QPQSQPLIPS PLQPQVAPPR PPAMDTTPSS KSQSPTELVS 1097 ASSSTSSSGK TSPTAPAVLS GPPTPPRQMQ SKQATPVSTP 1137 TRQVGSPKDP IPSDTAYETA SNIYEEIQED VPAPRHPPPA 1177 GPPPVIGAPM GPPPPLPNRR GPPPIPNRSG NAPPLPTRPA 1217 NN

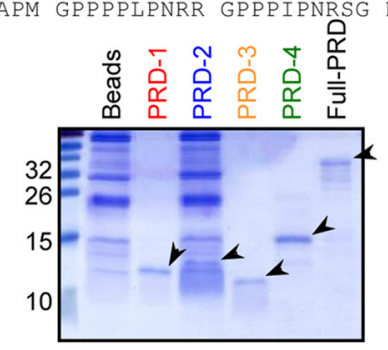

$\mathbf{F}$

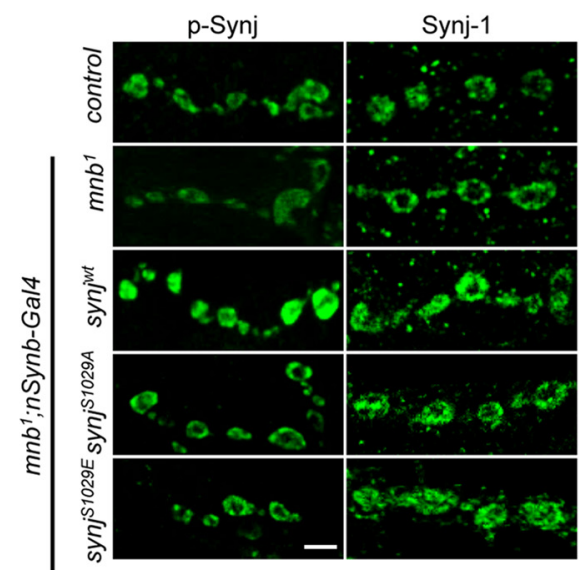

E

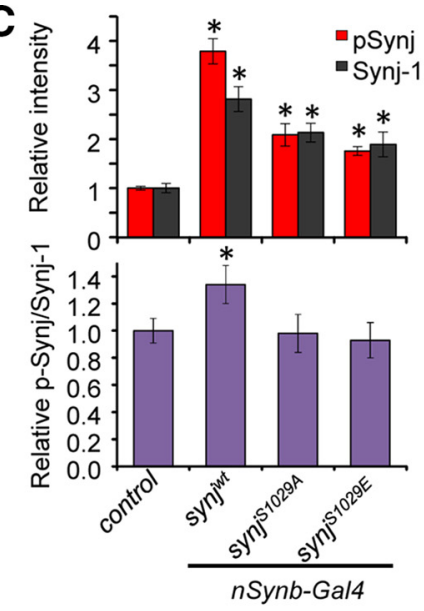

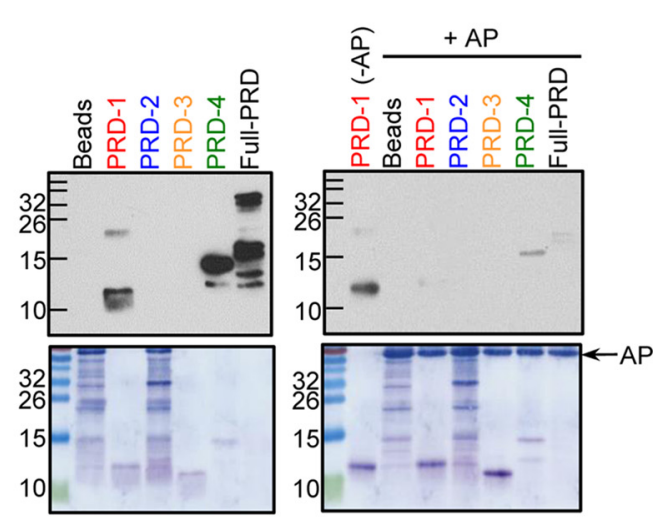

G

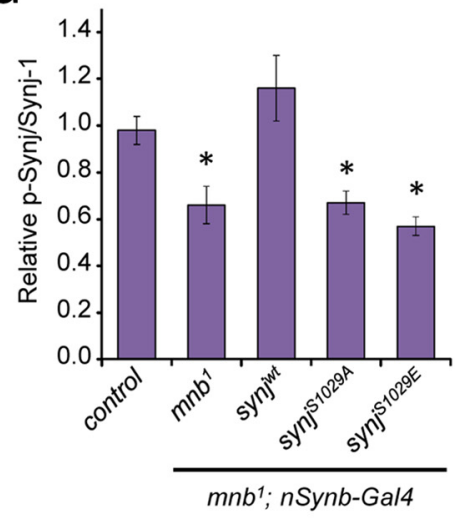

Figure 2. Phosphorylation of Synaptojanin at S1029 in vivo. A, Western blot of wild-type head protein extracts treated with and without alkaline phosphatase (AP) and detected using p-Synj ${ }^{51029}$ antibody and total Synj-1 antibody. B, Staining of Synj in the third intar NMJ using p-Synj and Synj-1 antibodies for the indicated genotypes in wild-type background. Scale bar, $5 \mu \mathrm{m}$. C, Quantification of relative staining intensity for p-Synj and Synj signals normalized to control (top), and the relative p-Synj/Synj signals. Error propagation was used to calculate fold change and SE. $n>12$ NMJ per genotype from at least 4 larvae per genotype. D, Different Synj PRD constructs are indicated by different color. Lower gel represents Coomassie staining of His-PRD constructs purified from bacteria. Lane labeled "Beads" indicates negative control done in parallel using bacteria transformed with empty PET-15b vector backbone. Arrows indicate purified PRD proteins. Full-PRD indicates full-length Synj PRD. E, Top blots, Western blots detected with pSynj antibody. Bottom, Direct blue 71(DB71) staining of the nitrocellulose membrane, showing the amount of protein loaded in each lane. Right, p-Synj does not detect signals well following alkaline phosphatase (AP) treatment, confirming the antibody is sensitive to phosphorylation status of Synj. $\boldsymbol{F}$, Staining of p-Synj and Synj-1 antibodies of the third instar NMJ for the indicated genotypes in $m n b^{7}$ mutant background. Scale bar, $5 \mu \mathrm{m}$. G, Quantification of relative staining intensity for $p$-Synj/Synj signals. $n=11 \mathrm{NMJ}$ from $\geq 4$ animals per genotype. Data are mean \pm SEM. ${ }^{*} p<0.05$ compared with control. 


\section{Phosphorylation of Synaptojanin at S1029 enhances Synaptojanin activity and alters Syanptojanin interaction with endophilin}

Previous studies have shown that Synj is a phosphoinositol phosphatase capable of regulating $\mathrm{PI}(4,5) \mathrm{P}_{2}$ levels (McPherson et al., 1996; Lee et al., 2004). To determine whether phosphorylation of Synj at S1029 regulates its enzymatic activity, we measured the 5 ' -phosphatase activity of immunoprecipitated Synj protein as assayed by conversion of BODIPY-labeled PI(4,5) $\mathrm{P}_{2}$ to BODIPY-PI(4)P using TLC (Fig. 3A). Consistent with previous results, addition of Mnb enhanced Synj ${ }^{\mathrm{wt}}$ activity, although it failed to enhance the $5^{\prime}$ phosphatase activity of Synj ${ }^{\text {S1029A }}$ or Synj ${ }^{\text {S1029E }}$ constructs (Fig. 3A). Interestingly, immunoprecipiated Synj ${ }^{\text {S1029E }}$ displayed higher $5^{\prime}$ phosphatase activity compared with Synj ${ }^{\text {wt }}$ construct, suggesting that phosphorylation at this site is necessary and sufficient to enhance Synj enzymatic activity. Furthermore, we tested the importance of Synj phosphorylation at S1029 in regulating its interaction with endophilin, an endocytic protein thought to recruit Synj to the synapse (Fig. 3B) (Schuske et al., 2003; Verstreken et al., 2003). We found that the phospho-null Synj ${ }^{\text {S1029A }}$ construct displayed increased interaction with endophilin, whereas the phospho-mimetic Synj ${ }^{\text {S1029E }}$ construct showed reduced interaction (Fig. 3B). These results are consistent with our previous findings that $m n b^{1}$ mutants show elevated Synj-endophilin interaction and decreased Synj activity (Chen et al., 2014). Together, these results reveal that phosphorylation of Synj at S1029 is sufficient to alter both Synj phosphatase activity and regulate Synj interaction with endophilin.

To confirm whether phosphorylation of Synj is sufficient to alter Synj activity locally at the synapse in vivo, the EGFP fusion protein containing the phospholipase $\mathrm{C} \delta 1$ pleckstrin homology domain

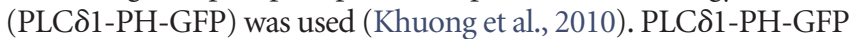
has been shown to specifically bind to $\mathrm{PI}(4,5) \mathrm{P}_{2}$, so that the fluorescence intensity in the synapse directly reflects the level of $\mathrm{PI}(4,5) \mathrm{P}_{2}$. Indeed, PLC $\delta 1-\mathrm{PH}-\mathrm{GFP}$ signal was elevated in $\operatorname{synj}^{1} / \mathrm{synj}^{2}$ mutant (here after referred to as synj mutant), confirming reduced Synj activity (Fig. 3C,D). Expression of synj ${ }^{w t}$ in synj mutant restored PLC 1 1-PH-GFP signal to control level, whereas expression of Synj ${ }^{\text {S1029A }}$ did not. Furthermore, expression of phospho-mimetic Synj ${ }^{\text {S1029E }}$ in synj mutant led to reduced PLC $81-\mathrm{PH}-\mathrm{GFP}$ signal locally within the synapse compared with the control, consistent with our biochemical data that phosphorylation of Synj at S1029 is sufficient to elevate Synj activity. This lack of rescue is not due to differential Synj expression because Western blot revealed that the levels of transgene expression as detected by HA and Synj antibodies are the same for different Synj constructs in synj mutant background (Fig. $3 E ; p>0.20)$. To further ensure that the levels of Synj protein present at the $\mathrm{NMJ}$ is the same across different transgenic lines, we compared the total Synj and p-Synj levels at the NMJ. We found that the levels of Synj at the synapse were not statistically different between the different transgenic lines, and both Synj ${ }^{\mathrm{S} 1029 \mathrm{~A}}$ and Synj ${ }^{\mathrm{S} 1029 \mathrm{E}}$ constructs showed lower levels of p-Synj compared with Synj ${ }^{\text {wt }}$, confirming that the antibody is sensitive to phosphorylation at S1029 (Fig. $3 F$ ). Together, these results indicate that phosphorylation of Synj alters Synj phosphatase activity in vivo.

We also noticed that synj mutants showed a decrease in the number of animals that survived to third instar larval stage (30\% viability of control; see Materials and Methods), consistent with a previous report (Verstreken et al., 2003). We found that expression of synj ${ }^{w t}$ in synj mutant background restored viability of synj mutant to $100 \%$, but expression of either Synj ${ }^{\text {S1029A }}$ or Synj ${ }^{\text {S1029E }}$ only partially rescued survival of synj mutant $(71 \%$ and $50 \%$ of control for Synj ${ }^{\mathrm{S} 1029 \mathrm{~A}}$ and Synj ${ }^{\mathrm{S} 1029 \mathrm{E}}$, respectively). These data suggest that dynamic phosphorylation of Synj is important dur- ing development, perhaps by regulating proper phosphatase activity or by modulating protein interactions with the right partner during development.

\section{Phosphorylation of Synaptojanin at S1029 is required for endocytosis but is not necessary for reliable neurotransmission at high stimulation frequency}

Synj is known to play an important role in synaptic vesicle recycling (Cremona et al., 1999; Schuske et al., 2003; Verstreken et al., 2003; Dickman et al., 2005; Mani et al., 2007). To further understand the impact of Synj phosphorylation in regulating synaptic vesicle endocytosis, we measured the ability of wild-type, phospho-null, and phospho-mimetic Synj constructs in rescuing the endocytosis defects of synj mutant. To this end, we used FM1-43 dye labeling, a lipophilic dye that can incorporate into synaptic vesicles during endocytosis. Compared with control, stimulation of synj mutant for 5 min using high extracellular potassium $(60 \mathrm{~mm})$ resulted in a significantly reduced FM1-43 loading (Fig. $4 A, B$ ). Expression of $s y n j^{w \mathrm{t}}$ or $s y n j{ }^{\mathrm{S} 1029 E}$ construct in synj mutant rescued FM1-43 load, but the phospho-null synj ${ }^{\text {S1029A }}$ construct did not. Because FM1-43 uptake is a function of both endocytosis and exocytosis, we further determined whether phospho-defective Synj ${ }^{\text {S1029A }}$ influences exocytosis by calculating the amount of FM1-43 that was released following subsequent stimulation, and then normalized it to the total load. As seen in Figure $4 C$, there was no difference in FM1- $43 \Delta \mathrm{F} / \mathrm{F}_{\text {load }}$ across genotypes, suggesting that phosphorylation of Synj does not influence synaptic vesicle exocytosis but is important for active endocytosis of synaptic vesicles. Furthermore, this result indicates that dephosphorylated Synj ${ }^{\mathrm{S} 1029 \mathrm{~A}}$ is defective in the endocytosis of the active recycling pool.

Robust synaptic vesicle endocytosis is particularly important for maintaining synaptic transmission during high stimulation frequencies. We therefore used electrophysiological recordings to assay whether phosphorylation of Synj differentially affects sustained synaptic communication. Electrophysiology recordings indicate that the normal evoked EPSP amplitude was comparable across genotypes (Fig. 5A), consistent with normal exocytosis in different genotypes. Stimulation of synj mutant at high frequency $(10 \mathrm{~Hz})$ for a prolonged period $(10 \mathrm{~min})$ caused a fast rundown of EPSP, characteristic of endocytic mutants (Fig. 5B,C). Expression of Synj ${ }^{\mathrm{wt}}$ and Synj ${ }^{\mathrm{S} 1029 \mathrm{E}}$ in synj mutant background rescued the fast rundown phenotype of synj mutant, consistent with the FM1-43 loading data. Surprisingly, despite a substantial defect in FM1-43 load, expression of Synj ${ }^{\text {S1029A }}$ in synj mutant background also restored synaptic transmission to normal (Fig. 5C).

\section{Synaptojanin is required to maintain synaptic vesicle pool size}

The observation that synaptic transmission is maintained at high stimulation frequency despite a substantial defect in endocytosis as revealed by FM1-43 loading is intriguing and unexpected for Drosophila mutants with problems in synaptic vesicle endocytosis. There are at least two functionally distinct synaptic vesicle pools at the Drosophila NMJ: the ECP that is activated by high potassium or low-frequency stimulation; and the RP that is poorly accessed at low frequency but efficiently recruited by highfrequency stimulation at $\geq 10 \mathrm{~Hz}$ (Kuromi and Kidokoro, 1998, 2000; Kim et al., 2009). Defects in maintaining either pool size via endocytosis or in recruitment of these vesicle pools can affect synaptic transmission (Kuromi and Kidokoro, 1998, 2002; Verstreken et al., 2005). Because our FM1-43 experiment done using high potassium loading condition revealed that phosphorylation 
A

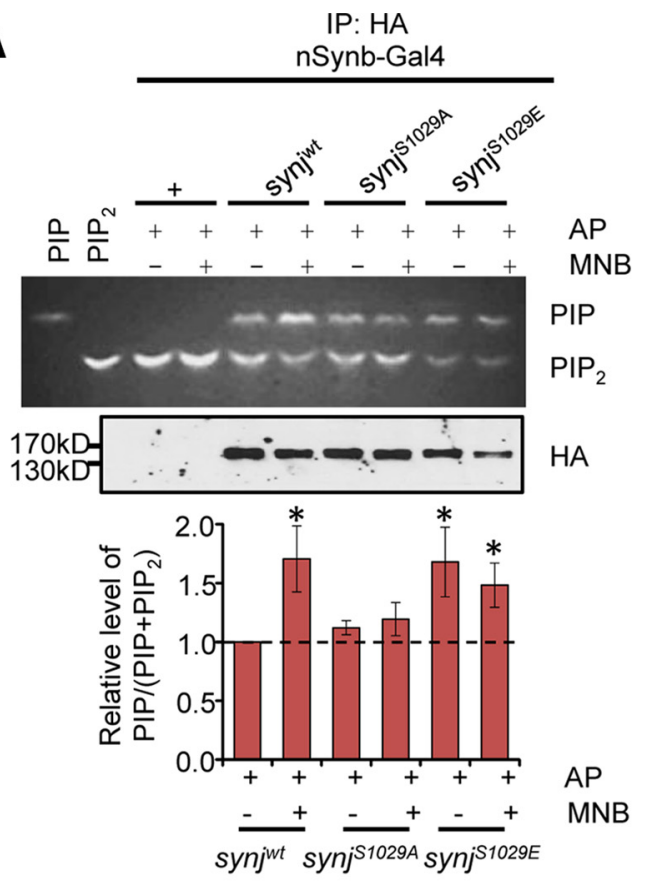

C

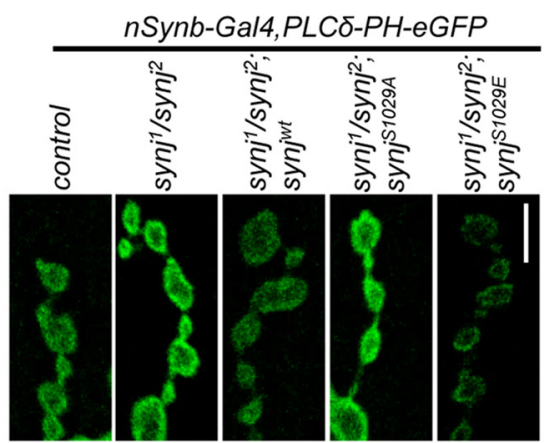

$\mathbf{E}$

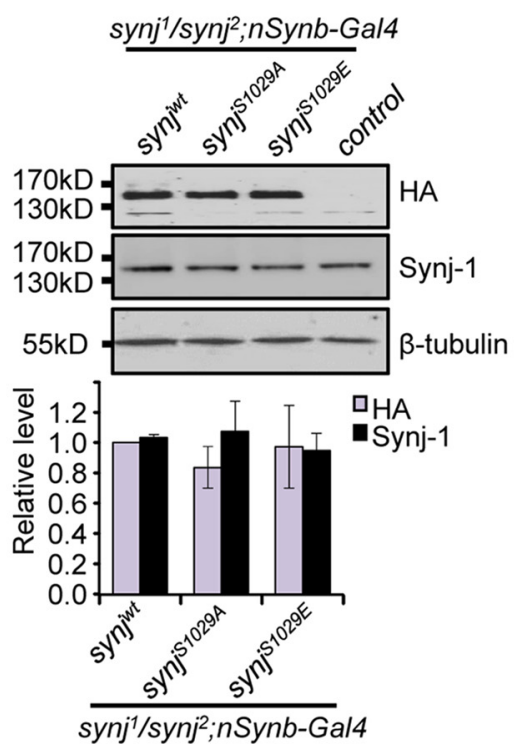

B

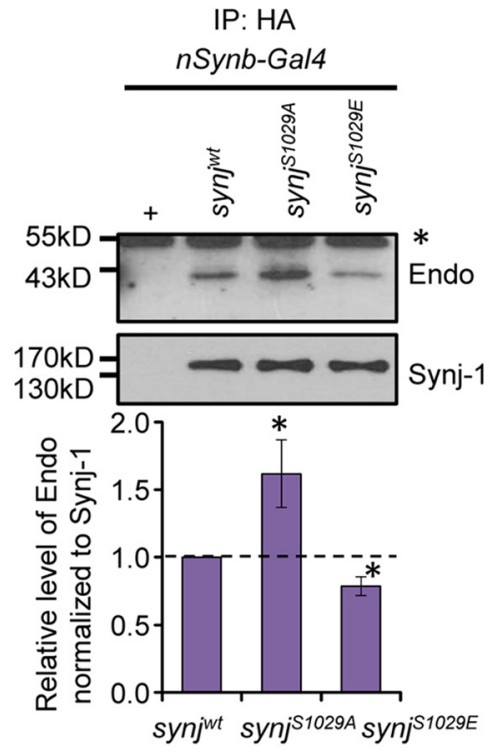

D

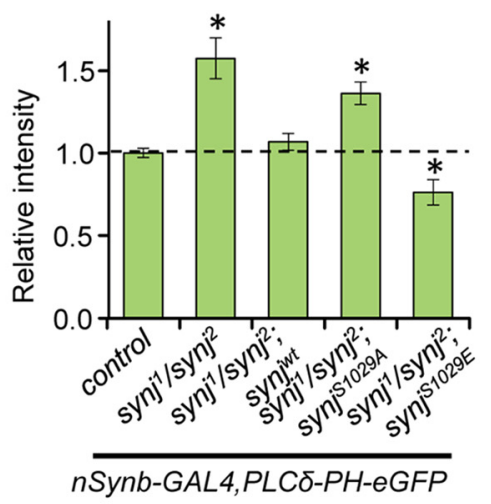

$\mathbf{F}$

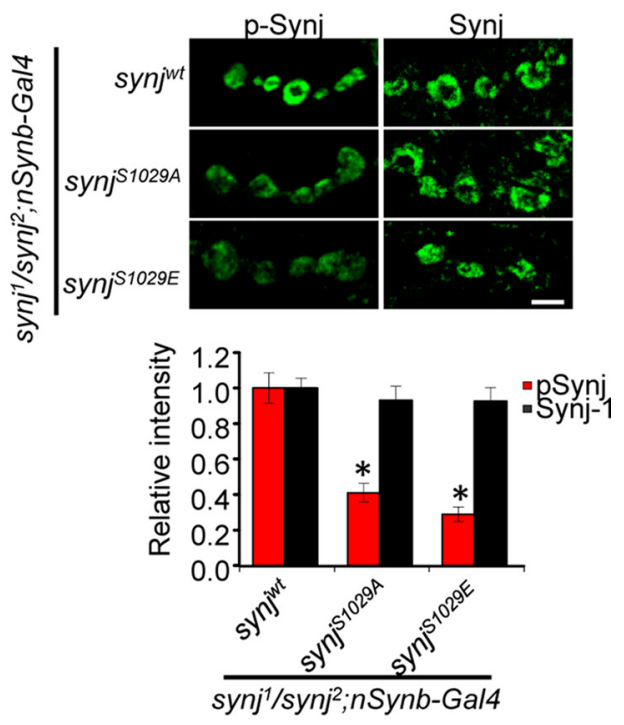

Figure 3. Phosphorylation of Synaptojanin at S1029 enhances Synaptojanin phosphoinositide phosphatase activity and alters its interaction with endophilin. $\boldsymbol{A}$, TLC showing conversion of BODIPY-PIP 2 to BODIPY-PIP by Synj constructs. Western blot represents levels of total Synj. Graph represents quantification of relative PIP to total phospholipid $\left(P I P+P I P_{2}\right)$ level. $n=8$ independent experiments per genotype per condition. B, Immunoprecipitation (IP) experiment using flies overexpressing Synj tagged with HA reveals that Synj ${ }^{\text {S1029A }}$ has strong interaction with Endophilin. *Background band due to lgG. Graph represents quantification of relative levels of endophilin-Synj interaction. $n=7$ independent experiments. Values have been normalized to total Synj and then fold change calculated relative to control. C, PIP ${ }_{2}$ levels in the NMJ measured by PLC $\delta$-PH-GFP for the indicated genotypes. D, Quantification of PIP ${ }_{2}$ levels (Figure legend continues.) 
A
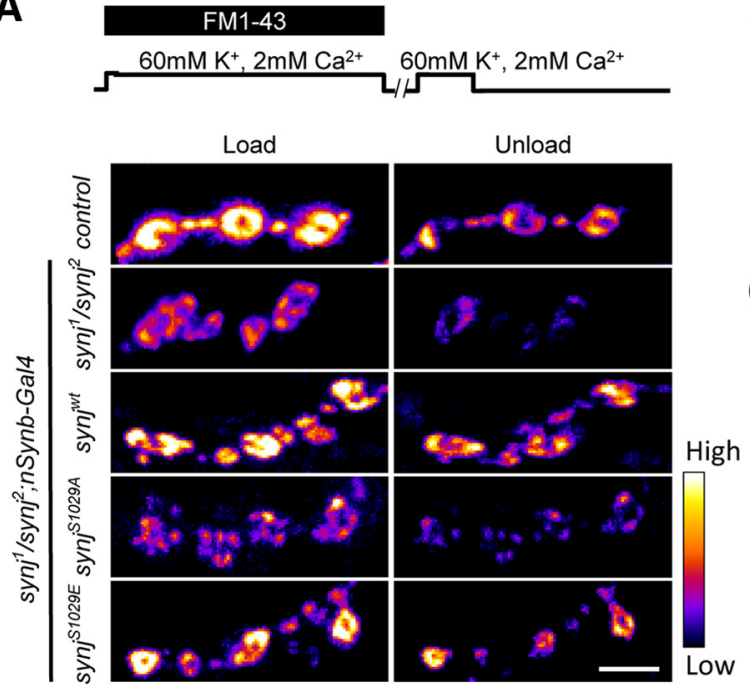

B

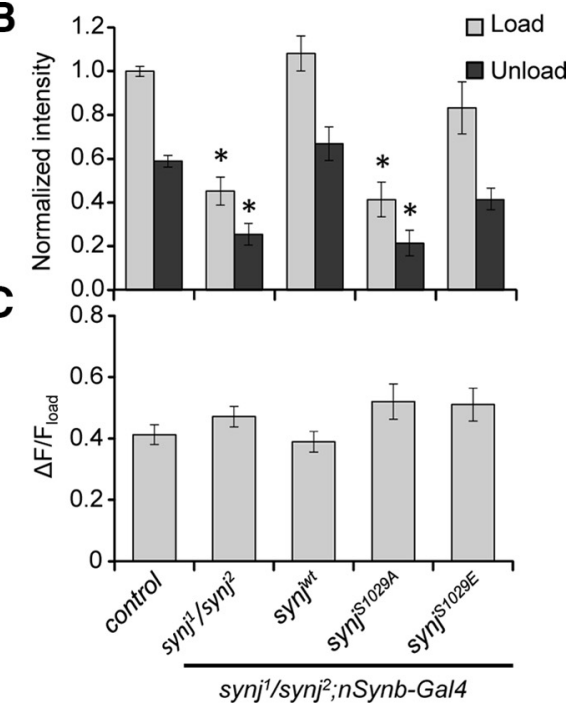

Figure 4. Phosphorylation of synaptojanin at S1029 is required for normal synaptic vesicle endocytosis. $\boldsymbol{A}$, Representative images of the NMJs after FM1-43 loading and unloading for the indicated genotypes in synj ${ }^{1} /$ synj $^{2}$ background. The $60 \mathrm{~mm} \mathrm{~K}^{+}$stimulation for 5 min was used to load the dye, and 1 min stimulation with $60 \mathrm{~mm} \mathrm{~K}{ }^{+}$was used to unload FM1-43. Scale bar, $5 \mu \mathrm{m}$. $\boldsymbol{B}$, Quantification of relative load and unload intensity for FM1-43 dye with normalization to loading intensity of the control. $\boldsymbol{C}$, Quantification of FM1-43 signal removed during unloading normalized to amount of FM1-43 loading. $n \geq 7$ NMJ from $n \geq 7$ larvae per genotype. Data are mean \pm SEM. ${ }^{*} p<0.05$ compared with control of the same condition.

of Synj at S1029 is required for endocytosis of the ECP vesicles, the fact that we did not observe a neurotransmission problem in the phospho-null Synj ${ }^{\text {S1029A }}$ NMJ during $10 \mathrm{~Hz}$ electrical stimulation further suggests that compared with synj mutant, the dephosphorylated Synj may (1) harbor a larger ECP and/or RP vesicle pool size, and/or (2) enhance mobilization of vesicles from the RP to compensate for defect in ECP endocytosis. First, we asked whether phospho-null Synj ${ }^{\text {S1029A }}$ maintains stable synaptic transmission in synj mutant by increasing the size of the ECP vesicles. We used electrophysiology to address this question because previous studies suggested that the ECP and RP are intermixed in the synapse and that standard EM and FM1-43 cannot readily differentiate the distinct pools based on location of the vesicles and staining pattern, respectively (Akbergenova and Bykhovskaia, 2009; Denker et al., 2009). We used bafilomycin A1, a drug that blocks the refilling of vesicles with neurotransmitter (Roseth et al., 1995), to estimate the size of the ECP using lowfrequency synaptic stimulation similar to a protocol described earlier (Kim et al., 2009). Electrical stimulation at $3 \mathrm{~Hz}$ in the presence of bafilomycin A1 treatment resulted in depletion of the ECP at this low stimulation frequency (Fig. $6 A$ ). The initial phase of the decay in EPSP amplitude represents the release of the ECP vesicles, whereas the slower decay phase results from a slow mixing of vesicles between the RP and ECP. We find that expression of the phospho-null Synj ${ }^{\text {S1029A }}$ in synj mutant background showed a slower decline in relative EPSP amplitude compared with synj mutant, suggesting that it has a larger ECP (Fig. 6A). To

$\leftarrow$

(Figure legend continued.) in the synapse. $n>12 \mathrm{NMJ}$ from $\geq 4$ larvae per genotype. Data are mean $\pm S E M .{ }^{*} p \leq 0.05$. $E$, Representative Western blots showing similar levels of transgene expression in synj mutant background detected with the indicated antibodies. Graph represents quantification of relative protein levels for the indicated lines normalized to $\beta$-tubulin as loading control. For HA staining, the level was normalized to synj ${ }^{w t}$ in synj mutant background because control flies do not express HA. For total Synj signal, the level was normalized to control flies (data not shown because the level was set to 1). $n=4$ independent experiments. $\boldsymbol{F}$, Staining of the third intar NMJ using p-Synj and Synj-1 antibodies for the indicated genotypes in synj mutant background. Scale bar, $5 \mu \mathrm{m}$. Quantification of the levels of Synj and $p$-Synj at the NMJ is graphed. $n \geq 12 \mathrm{NMJ}$ from at least 4 larvae per genotype. estimate the size of the ECP, we plotted the cumulative quantal content and used linear regression to determine the size of the ECP (Fig. 6A, B) (Delgado et al., 2000; Kim et al., 2009). We find that, compared with control, synj mutant showed a substantially reduced ECP, revealing that Synj is crucial for the maintenance of the size of the ECP (Fig. 6C). Expression of synj ${ }^{w \mathrm{t}}$, phospho-null synj $j^{S 1029 A}$ in synj mutant background all restored the size of the ECP. Interestingly, expression of the phospho-mimetic synj ${ }^{S 1029 E}$ in synj mutant background showed a small but significant increase in the estimated ECP size compared with control, although not compared with synj $j^{w \mathrm{t}}$ expression in synj mutant background (Fig. 6C). Together, these results thus imply that the phosphonull Synj ${ }^{\text {S1029A }}$ rescues synaptic transmission of synj mutant by restoring ECP size to compensate for endocytic defect.

We also determined the total vesicle pool size by depleting synaptic vesicles at $10 \mathrm{~Hz}$ stimulation frequency in the presence of bafilomycin A1 and measured the cumulative quantal content. We found that synj mutants showed a substantially reduced total synaptic vesicle pool size compared with control (Fig. 6D). This suggests that Synj is required to maintain both the ECP and RP vesicle pool size, presumably through its ability to affect vesicle endocytosis. This result is consistent with previous EM studies demonstrating depletion of synaptic vesicles in the synaptic boutons of synj animals (Verstreken et al., 2003; Dickman et al., 2005). We also found that expression of different synj constructs all rescued the total number of synaptic vesicle pools to normal (Fig. 6D), suggesting that the phosphorylation status of Synj does not regulate total synaptic vesicle pool size. Interestingly, because the total pool size is a sum of the ECP and RP, the slightly larger ECP size observed for phospho-mimetic Synj ${ }^{\mathrm{S} 1029 \mathrm{E}}$ further indicates that it may have a reduced RP size compared with control.

Next, we tested whether altered mobilization of vesicles from the RP contributes to the rescue of synj mutant by phospho-null synj ${ }^{\text {S1029A }}$ expression. To this end, we blocked RP mobilization by inhibiting the myosin light chain kinase activity (Mochida et al., 1994; Ryan, 1999). Incubating control NMJ with ML-7, which has previously been shown to block myosin light chain kinase and RP mobilization (Verstreken et al., 2005; Kim et al., 2009), caused 
A

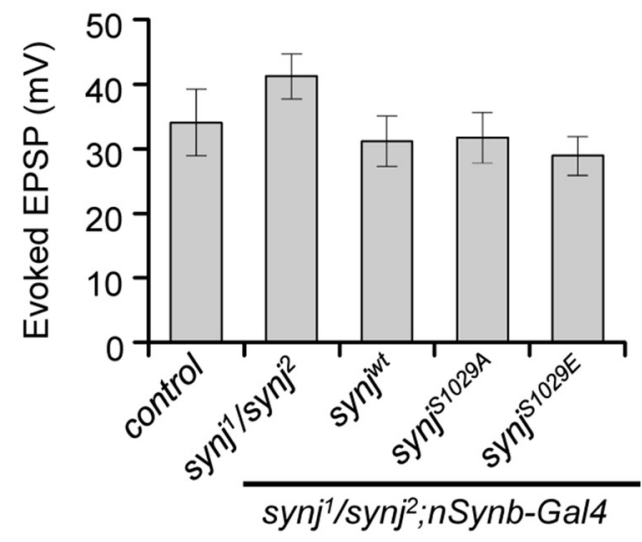

C

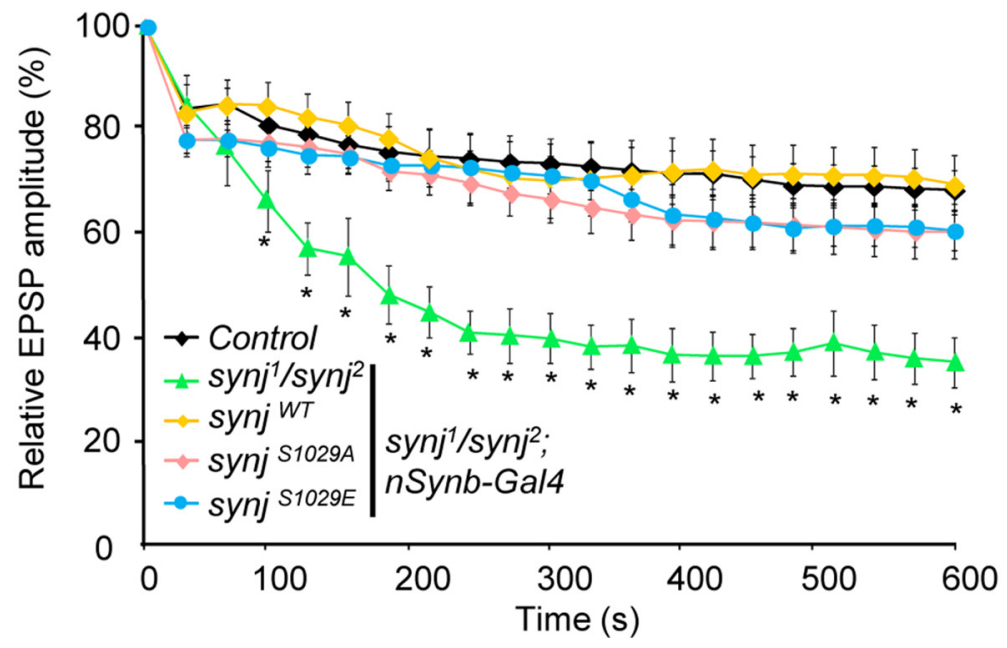

Figure 5. Expression of either phospho-null or phospho-mimetic Synaptojanin can rescue neurotransmission defect of synj mutant at high stimulation frequency. $\boldsymbol{A}$, Average evoked EPSP recorded using $\mathrm{HL}-3$ containing $0.4 \mathrm{~mm} \mathrm{Ca^{2+ }} . n=6 \mathrm{NMJ}$ from 6 larvae per genotype. $\boldsymbol{B}$, Representative EPSP recordings during $10 \mathrm{~Hz}$ stimulation for $10 \mathrm{~min}$ in $\mathrm{HL}-3$ containing $2 \mathrm{~mm} \mathrm{Ca}^{2+} . \boldsymbol{C}$, Relative EPSP amplitude plotted over time for the indicated genotypes. $n>6$ NMJ from $>6$ larvae for each genotype. ${ }^{*} p<0.05$ compared with control. Data are mean \pm SEM.

a more rapid decline in evoked EPSP during high-frequency stimulation compared with NMJ incubated with vehicle control (Fig. 6E). We reasoned that, if Synj ${ }^{\text {S1029A }}$ does indeed rescue synaptic transmission of synj mutant by facilitating RP mobilization, inhibiting RP recruitment would abolish its ability to restore synaptic transmission during high-frequency stimulation to become the synj mutant phenotype. We found that inhibiting RP mobilization in Synj ${ }^{\text {S1029A }}$ flies still rescued synaptic transmission to control level during the early phase of stimulation but caused a more rapid decline in EPSP amplitude so that it no longer rescued the synj mutant phenotype toward the later phase of stimulation (Fig. 6E). Because the RP is thought to be recruited following depletion of the readily releasable pool and the recycling pool (ECP), our results support the claim that Synj ${ }^{\text {S1029A }}$ can rescue ECP size in synj mutant, and further indicate that they rely on mobilization of the RP during persistent stimulation conditions to maintain stable synaptic transmission in the presence ECPrecycling defects. Interestingly, consistent with the observation that Synj ${ }^{\text {S1029E }}$ has a larger ECP size, Synj ${ }^{\text {S1029E }}$ maintained synaptic transmission a little better than control for a short duration in the absence of RP mobilization (Fig. 6E).
B

$10 \mathrm{~Hz}, 10^{\prime}$

\section{Control}
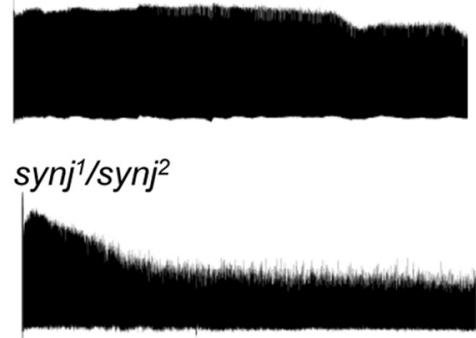

synj $1 /$ synj 2 ; nSynb-Gal4/synj $1029 A$

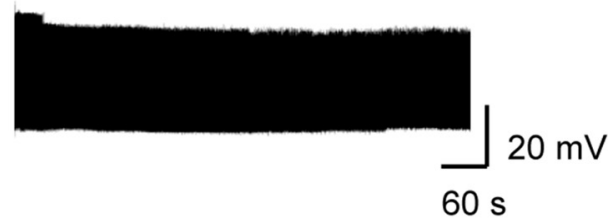

\section{Phosphorylation status of Synj differentially affects the} recycling of ECP and RP

Our data indicate that Synj is required to maintain the vesicle pool size, but how does phospho-null Synj ${ }^{\text {S1029A }}$, which is defective in ECP endocytosis, rescue the vesicle pool size? We hypothesized that dephosphorylated Synj may still retrieve vesicles through a slower pathway, such as endocytosis of the RP, which has been shown to occur at the cessation of stimulation, so that vesicles could eventually replenish both the RP and the ECP. To this end, we took advantage of an established FM1-43 labeling paradigm to label both the ECP and RP by including FM1-43 both during $10 \mathrm{~min}$ of $10 \mathrm{~Hz}$ electrical stimulation and $5 \mathrm{~min}$ after stimulation (Verstreken et al., 2008). Relative RP vesicle endocytosis was then determined by unloading the ECP with high $\mathrm{KCl}$. FM1-43 load thus represents the amount of both ECP + RP endocytosis, whereas FM1-43 signal remaining after unload with high $\mathrm{KCl}$ represents endocytosis of the RP. We found that, compared with control, synj mutant showed a substantial decrease in both FM1-43 load and FM1-43 retention following ECP unloading, suggesting that Synj protein is crucial for ECP and RP endocytosis (Fig. 7A). This claim is supported by observation that 
A

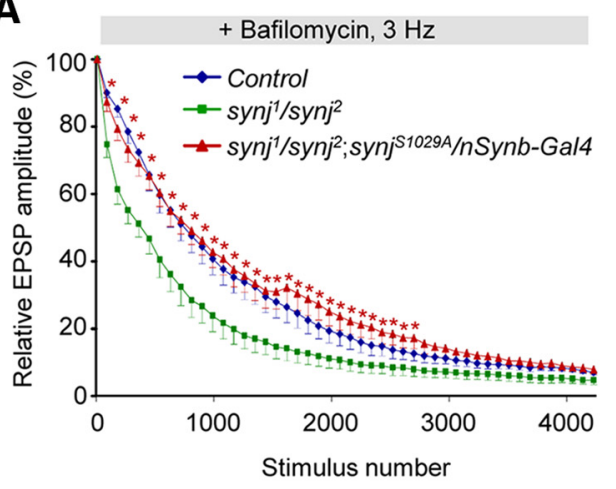

C

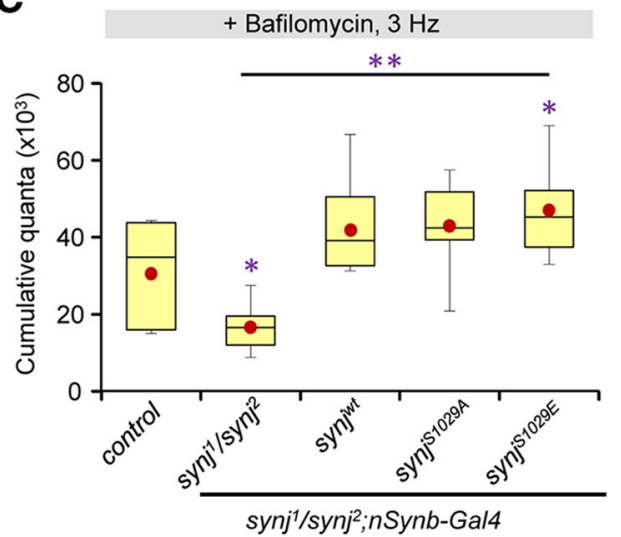

B

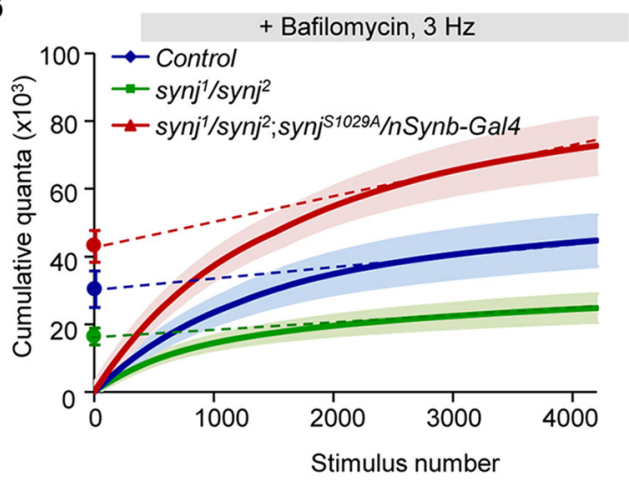

D

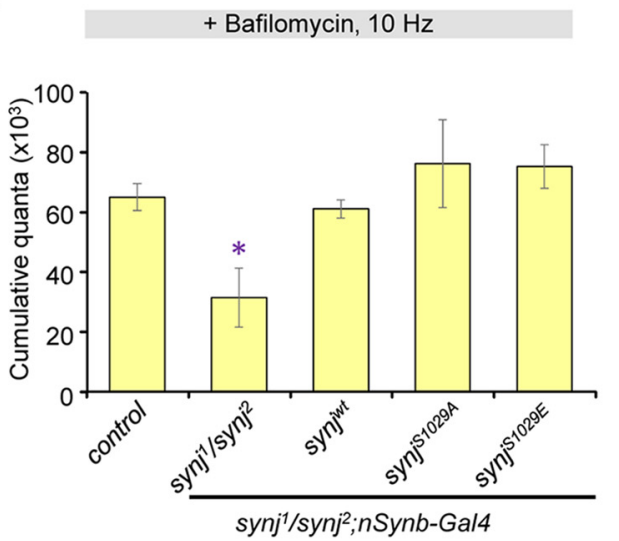

\section{$\mathbf{E}$}

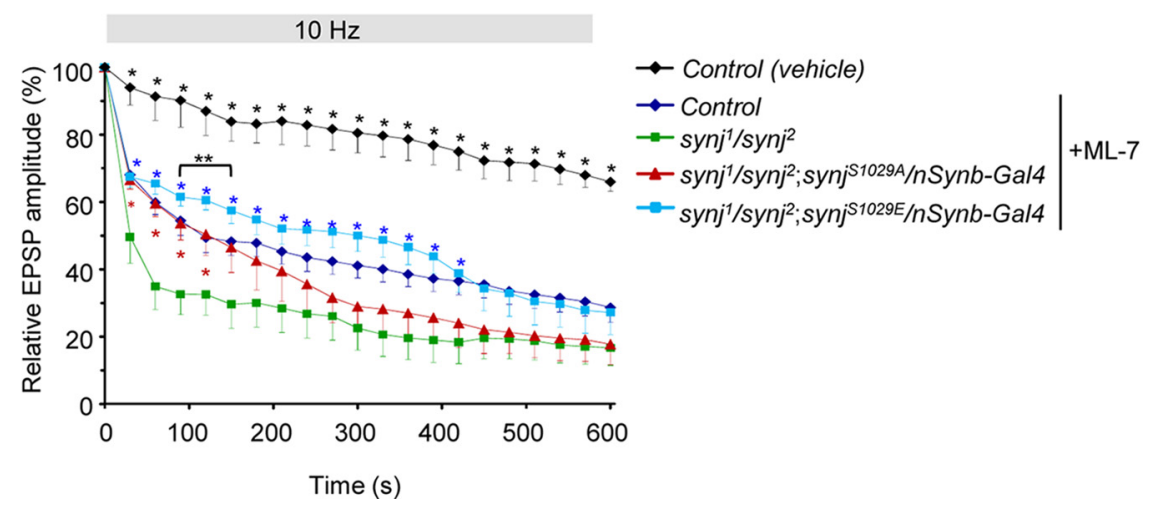

Figure 6. Phosphorylation of Synaptojanin differentially affects the size of the ECP and RP. $A$, Relative EPSP amplitude plotted over time for indicated genotypes in the presence of $2 \mu \mathrm{m}$ bafilomycin A1 with $3 \mathrm{~Hz}$ stimulation. Expression of the phospho-null Synj ${ }^{1029 A}$ restores the size of the ECP. Data are mean \pm SEM. ${ }^{*} p<0.05$, comparing synj $1 /$ synj ${ }^{2}$ with phospho-null Synj ${ }^{\text {S1029A }}$. $B$, Cumulative quantal content plot. Linear regression analysis was used to back extrapolate from points between stimulus pulses 2500 and 4200 for indicated genotypes. ECP estimates were observed from the $y$-intercepts. C, Box plot showing ECP estimates obtained from linear regression analysis of cumulative quantal content plot for the indicated genotypes. Red dot indicates the mean value. ${ }^{*} p<0.05$, compared with control. ${ }^{* *} p<0.05$, indicated genotypes compared with synj ${ }^{1}$ synj ${ }^{2}$. $\boldsymbol{A}-\boldsymbol{C}, n=6 \mathrm{NMJ}$ from 6 larvae for each genotypes. $\boldsymbol{D}$, Total synaptic vesicle pool size estimates for the indicated genotypes. synj ${ }^{1} /$ synj $^{2}$ has a reduced total pool size. $n=4$ per genotype. Data are mean \pm SEM. ${ }^{*} p<0.05$ compared with control. $E$, Relative EPSP amplitude in the presence of ML-7 treatment (to inhibit mobilization of the RP vesicles) or vehicle control. $n>6 \mathrm{NMJ}$ from $>6$ larvae per genotype. Data are mean \pm SEM. ${ }^{*}$ (black), $p<0.05$, comparing control treated with vehicle (DMSO) to synj mutant. *(red), $p<0.05$, comparing synj ${ }^{51029 A}$ expression in synj mutant background to synj mutant. * (blue), $p<0.05$, comparing control or synj $51029 E$ expression in synj mutant background to synj mutant. ${ }^{* *}$ (and bracket), $p<0.05$, comparing control with Synj ${ }^{51029 \mathrm{E}}$ expression in synj mutant background. Nonlinear summation correction was used to determine quantal content.

Synj ${ }^{\mathrm{wt}}$ expression rescued both the endocytosis of the ECP and RP in synj mutant to normal (Fig. $7 A, B$ ). On the other hand, expression of phospho-null Synj ${ }^{\text {S1029A }}$ showed a reduced FM1-43 load but normal FM1-43 retention following ECP unload, suggesting that it has normal RP endocytosis but reduced ECP endocytosis. Interestingly, expression of phospho-mimetic Synj ${ }^{\mathrm{S} 1029 \mathrm{E}}$ restored FM1-43 load while displaying reduced FM1-43 dye retention following subsequent high potassium stimulation, indicating enhanced ECP endocytosis but reduced $\mathrm{RP}$ endocytosis. We also calculated the relative amount of RP endocytosis by normalizing FM1-43 staining remaining after ECP unloading to the total FM1-43 uptake (Fig. 7C). This calculation represents the proportion of vesicle retrieval that went through the RP route since they were not readily released by $\mathrm{KCl}$ depolarization. We found that phospho-mimetic Synj ${ }^{\mathrm{S} 1029 \mathrm{E}}$ flies have reduced RP endocytosis, whereas synj mutant did not alter 
A

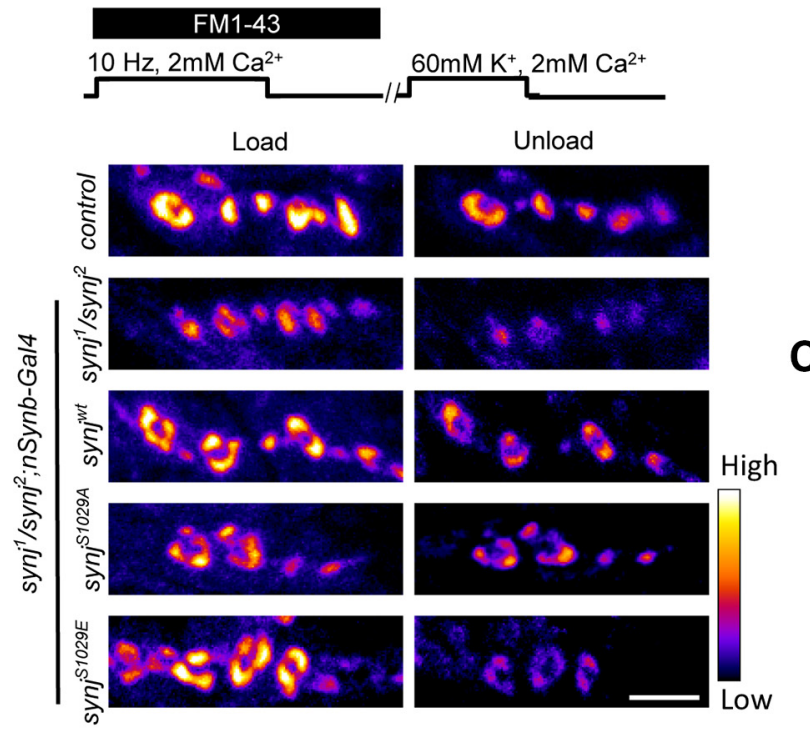

B
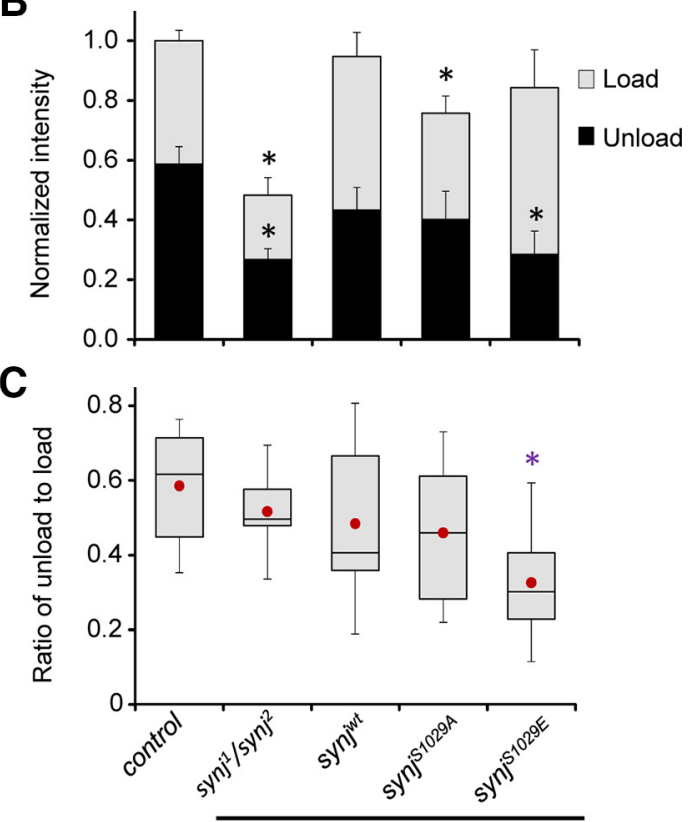

synj ${ }^{1} /$ synj $^{2} ; n$ Synb-Gal4
D

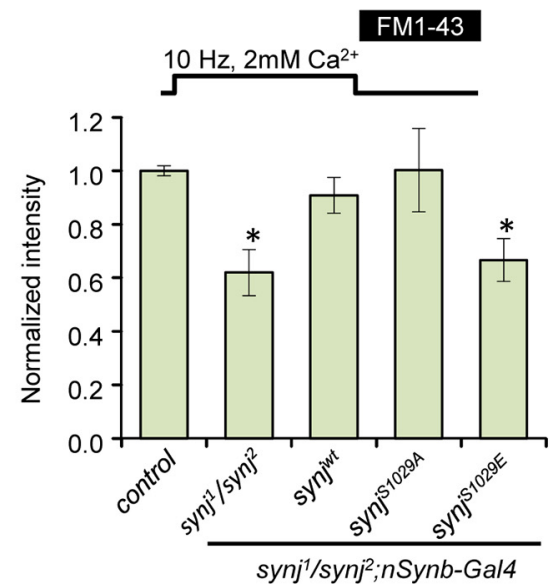

E

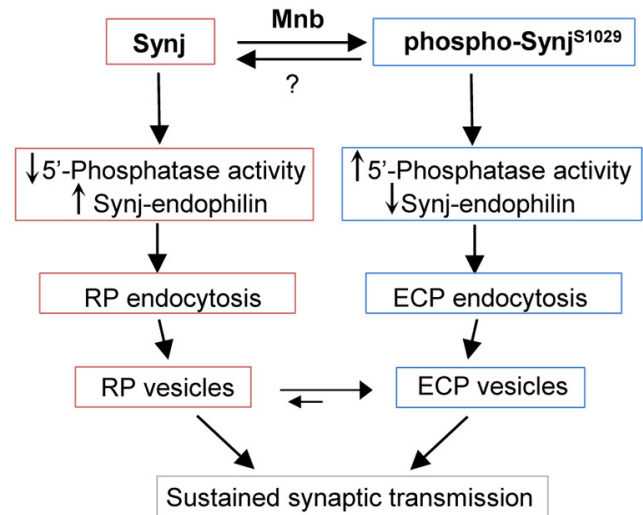

Figure 7. Phosphorylation of Synaptojanin affects endocytosis of the RP. A, Representative images of the NMJ after FM1-43 loading and unloading. The $10 \mathrm{~Hz}$ electrical stimulation for 10 min plus an additional 5 min waiting was used to load both the ECP and RP. A subsequent 5 min stimulation using $60 \mathrm{~mm} \mathrm{~K}^{+}$was used to unload the ECP, revealing the amount of RP endocytosis. Scale bar, $5 \mu \mathrm{m} . \boldsymbol{B}$, Relative FM1-43 loading and unloading intensities for indicated genotypes with normalization to the loading intensity of control. Data are mean \pm SEM. ${ }^{*} p<0.05$ compared with control of the same loading/unloading condition. C, Box plot showing the relative amount of RP endocytosis by normalizing FM1-43 dye remaining after ECP unloading to the total FM1-43 dye loading. Red dot indicates the mean. $n>6 \mathrm{NMJ}$ from $>6$ larvae per genotype. ${ }^{*} p<0.05$ compared with control. $\boldsymbol{D}$, Relative FM1-43 loading of the RP pool for the indicated genotypes. RP was loaded by adding in FM1-43 dye immediately following cessation of electrical stimulation. Data are mean \pm SEM. ${ }^{*} p<0.05$ compared with control. $n>5 \mathrm{NMJ}$ from $>5$ larvae per genotype. $E$, Model depicting recycling of distinct functional vesicle pools modulated by phosphorylated-Synj or dephosphorylated Synj.

the proportion that went through the RP pathway, likely because it was deficient in both RP and ECP endocytosis. In addition, because the RP is thought to be retrieved following stimulation (Kuromi and Kidokoro, 2002; Verstreken et al., 2005; Akbergenova and Bykhovskaia, 2009), we selectively monitored RP endocytosis by adding in FM1-43 dye immediately following $10 \mathrm{~min}$ of $10 \mathrm{~Hz}$ stimulation. Figure $7 D$ shows that synj mutants showed a defect in RP loading and Synj ${ }^{\mathrm{S} 1029 \mathrm{E}}$ failed to rescue this defect. On the other hand, expression of Synj ${ }^{\text {wt }}$ or Synj ${ }^{\text {S1029A }}$ in synj mutant background rescued RP endocytosis. These data not only confirm that Synj is important for RP endocytosis but further indicate that the phosphorylation status of Synj differentially regulates the endocytosis of different vesicle pools. Together, these results reveal that phosphorylated Synj preferentially participates in the endocytosis of the ECP at the expense of RP endocytosis, whereas dephosphorylated Synj has defective ECP endocytosis but maintains the endocytosis of the RP.

\section{Discussion}

In this study, we demonstrate that Mnb phosphorylates Synj at S1029 in vivo and that the phosphorylation status of Synj differentially regulates endocytosis of distinct functional synaptic vesicle pools at the Drosophila NMJ. We report that phosphorylation of Synj at S1029 enhances Synj 5' -phosphatase activity, reduces interaction between Synj and endophilin, and preferentially promotes the recycling of the ECP vesicles at the expense of RP recycling. On the other hand, dephosphorylated Synj maintains RP vesicle endocytosis but is defective in ECP vesicle endocytosis. Our data reveal a novel role 
for Synj in both ECP and RP vesicle endocytosis and in maintaining vesicle pool size, and suggests that coordinated phosphorylation and dephosphorylation of Synj during synaptic activity optimize endocytosis of different functional synaptic vesicle pools. Together with our previous data showing acute phosphorylation of Synj by the Mnb kinase during synaptic activity (Chen et al., 2014), we propose a simplified model in which synaptic activity triggers Mnb-dependent phosphorylation of Synj at S1029, which acts to selectively enhance ECP endocytosis during stimulation. Dephosphorylation of Synj at S1029 then promotes RP vesicle endocytosis (Fig. 7E). This precisely controlled phosphorylation-dephosphorylation cycle delicately controls the proper timing of the ECP and RP endocytosis to sustain communication across a wide range of synaptic activities.

How does Synj modulate endocytosis of both the ECP and RP vesicles? While some studies propose that newly reformed vesicles are recaptured randomly by different vesicle pools (Ryan and Smith, 1995; Schikorski and Stevens, 2001), others suggest that the ECP and RP vesicles are retrieved through different mechanisms (Koenig and Ikeda, 1996; Kuromi and Kidokoro, 2002). Our findings that the phosphorylation status of Synj differentially affected distinct synaptic vesicle pool recycling support the model that the ECP and RP are recycled through specific pathways. It is thought that the recycling of the ECP vesicles, which participate in synaptic vesicle release during moderate synaptic activity, is rapidly replenished by clathrin-mediated endocytosis (Rizzoli and Betz, 2005; Cheung et al., 2010). Synj has an established role in clathrin-mediated endocytosis. synj mutations in vertebrates and invertebrates lead to an elevation in $\mathrm{PI}(4,5) \mathrm{P}_{2}$ levels, accumulation of densely coated vesicles, and a delay in endocytic vesicle reavailability, suggesting that Synj functions in clathrin uncoating through dephosphorylation of $\mathrm{PI}(4,5) \mathrm{P}_{2}$ (Cremona et al., 1999; Harris et al., 2000; Kim et al., 2002; Verstreken et al., 2003; Dickman et al., 2005). Our observations that the phosphomimetic Synj ${ }^{\mathrm{S} 1029 \mathrm{E}}$ rescued ECP endocytosis of synj mutant while the dephosphorylated Synj did not, thus imply that phosphorylated Synj selectively participates in clathrin-mediated endocytosis. Previous reports have shown that the $5^{\prime}$-phosphatase domain of Synj is crucial for vesicle endocytosis (both for low- and highfrequency stimulation in hippocampal neurons), whereas interaction with endophilin is dispensable for clathrin-mediated endocytosis during mild activity (Granseth et al., 2006; Mani et al., 2007; Dong et al., 2015). These results thus suggest that the increase in $5^{\prime}$-phosphatase activity of phospho-memetic Synj ${ }^{\mathrm{S} 1029 \mathrm{E}}$, rather than altered interaction with endophilin, likely underlies its ability to rescue ECP endocytosis of synj mutants.

The RP vesicles, which are recruited only during intense neuronal activity, are thought to be selectively replenished by a bulk endocytosis pathway (Richards et al., 2000; Rizzoli and Betz, 2005; Clayton and Cousin, 2009; Cheung et al., 2010). The role that Synj plays in this pathway is not well understood, although recordings from hippocampal neurons of synj $j^{-1-}$ mutant suggest that Synj is required for all modes of synaptic retrieval, including bulk endocytosis (Mani et al., 2007). Using FM1-43 loading and unloading conditions that permit preferential labeling of RP vesicles, we confirmed that in addition to ECP endocytosis, synj is also important for normal RP endocytosis. This is further supported by our electrophysiological recordings demonstrating a smaller total vesicle pool size in synj mutant, as well as previous work showing an overall depletion of synaptic vesicles at the synapse as revealed by EM (Verstreken et al., 2003; Dickman et al., 2005). Although the molecular mechanisms differentially regulating ECP and RP endocytosis are not well understood, we anticipate that Synj interaction with endophilin will likely play an important role. Synj-endophilin interaction has been proposed to be particularly important for bulk or RP endocytosis because expression of Synj that cannot interact with endophilin display selective endocytic defects during intense but not mild neuronal activity (Mani et al., 2007; Clayton and Cousin, 2009). Interestingly, we also find that phosphorylated Synj, which shows reduced interaction with endophilin, displays decreased RP endocytosis. Conversely, dephosphorylated Synj, which display increased interaction with endophilin, maintains RP endocytosis while having a reduced $5^{\prime}$-phosphatase activity. We envision a scenario in which dephosphorylated Synj is preferentially recruited by endophilin, which leads to modification of the membrane phospholipids at a slower rate due to the lower enzymatic activity of the dephosphorylated Synj, thus providing a distinct temporal and spatial profile required for different synaptic vesicle retrieval modes. RP vesicles can then be regenerated from bulk endosomes (Richards et al., 2000; Rizzoli and Betz, 2005; Clayton and Cousin, 2009; Cheung et al., 2010; Cheung and Cousin, 2013; Wu et al., 2014), and mobilization of the RP can replenish the ECP vesicles as well as restore the size of vesicle pools to sustain synaptic transmission during high-frequency stimulation (Delgado et al., 2000; Kuromi and Kidokoro, 2000; Kidokoro et al., 2004). In line with this, we find that phospho-null Synj ${ }^{\text {S1029A }}$ becomes dependent on mobilization of RP during prolonged highfrequency stimulation. The exact role of Synj in RP/bulk endocytosis and RP formation/mobilization, as well as the molecular mechanisms differentially regulating clathrin-mediated and bulk endocytosis, remain interesting areas to investigate in the future. In addition, as perturbations in Synj and Mnb have been linked to neurological disorders, including Parkinson's, autism, and Down syndrome (Guimera et al., 1999; Arai et al., 2002; Dowjat et al., 2007; O'Roak et al., 2012; Krebs et al., 2013), understanding mechanisms modulating Mnb-dependent phosphorylation of Synj and identifying phosphatase(s) that trigger dephosphorylation of Synj will be important topics to study in the future.

\section{References}

Adayev T, Chen-Hwang MC, Murakami N, Wang R, Hwang YW (2006) MNB/DYRK1A phosphorylation regulates the interactions of synaptojanin 1 with endocytic accessory proteins. Biochem Biophys Res Commun 351:1060-1065. CrossRef Medline

Akbergenova Y, Bykhovskaia M (2009) Stimulation-induced formation of the reserve pool of vesicles in Drosophila motor boutons. J Neurophysiol 101:2423-2433. CrossRef Medline

Arai Y, Ijuin T, Takenawa T, Becker LE, Takashima S (2002) Excessive expression of synaptojanin in brains with Down syndrome. Brain Dev 24: 67-72. CrossRef Medline

Chen CK, Bregere C, Paluch J, Lu JF, Dickman DK, Chang KT (2014) Activity-dependent facilitation of Synaptojanin and synaptic vesicle recycling by the Minibrain kinase. Nat Commun 5:4246. CrossRef Medline

Cheung G, Cousin MA (2013) Synaptic vesicle generation from activitydependent bulk endosomes requires calcium and calcineurin. J Neurosci 33:3370-3379. CrossRef Medline

Cheung G, Jupp OJ, Cousin MA (2010) Activity-dependent bulk endocytosis and clathrin-dependent endocytosis replenish specific synaptic vesicle pools in central nerve terminals. J Neurosci 30:8151-8161. CrossRef Medline

Clayton EL, Cousin MA (2009) The molecular physiology of activitydependent bulk endocytosis of synaptic vesicles. J Neurochem 111: 901-914. CrossRef Medline

Cremona O, Di Paolo G, Wenk MR, Lüthi A, Kim WT, Takei K, Daniell L, Nemoto Y, Shears SB, Flavell RA, McCormick DA, De Camilli P (1999) Essential role of phosphoinositide metabolism in synaptic vesicle recycling. Cell 99:179-188. CrossRef Medline

Delgado R, Maureira C, Oliva C, Kidokoro Y, Labarca P (2000) Size of vesicle pools, rates of mobilization, and recycling at neuromuscular synapses of a Drosophila mutant, shibire. Neuron 28:941-953. CrossRef Medline

Denker A, Kröhnert K, Rizzoli SO (2009) Revisiting synaptic vesicle pool 
localization in the Drosophila neuromuscular junction. J Physiol 587: 2919-2926. CrossRef Medline

Dickman DK, Horne JA, Meinertzhagen IA, Schwarz TL (2005) A slowed classical pathway rather than kiss-and-run mediates endocytosis at synapses lacking synaptojanin and endophilin. Cell 123:521-533. CrossRef Medline

Dong Y, Gou Y, Li Y, Liu Y, Bai J (2015) Synaptojanin cooperates in vivo with endophilin through an unexpected mechanism. eLife 4.

Dowjat WK, Adayev T, Kuchna I, Nowicki K, Palminiello S, Hwang YW, Wegiel J (2007) Trisomy-driven overexpression of DYRK1A kinase in the brain of subjects with Down syndrome. Neurosci Lett 413:77-81. CrossRef Medline

Granseth B, Odermatt B, Royle SJ, Lagnado L (2006) Clathrin-mediated endocytosis is the dominant mechanism of vesicle retrieval at hippocampal synapses. Neuron 51:773-786. CrossRef Medline

Guimera J, Casas C, Estivill X, Pritchard M (1999) Human minibrain homologue (MNBH/DYRK1): characterization, alternative splicing, differential tissue expression, and overexpression in Down syndrome. Genomics 57:407-418. CrossRef Medline

Haffner C, Di Paolo G, Rosenthal JA, de Camilli P (2000) Direct interaction of the $170 \mathrm{kDa}$ isoform of synaptojanin 1 with clathrin and with the clathrin adaptor AP-2. Curr Biol 10:471-474. CrossRef Medline

Harris TW, Hartwieg E, Horvitz HR, Jorgensen EM (2000) Mutations in synaptojanin disrupt synaptic vesicle recycling. J Cell Biol 150:589-600. CrossRef Medline

Himpel S, Tegge W, Frank R, Leder S, Joost HG, Becker W (2000) Specificity determinants of substrate recognition by the protein kinase DYRK1A. J Biol Chem 275:2431-2438. CrossRef Medline

Iossifov I, Ronemus M, Levy D, Wang Z, Hakker I, Rosenbaum J, Yamrom B, Lee YH, Narzisi G, Leotta A, Kendall J, Grabowska E, Ma B, Marks S, Rodgers L, Stepansky A, Troge J, Andrews P, Bekritsky M, Pradhan K, et al. (2012) De novo gene disruptions in children on the autistic spectrum. Neuron 74:285-299. CrossRef Medline

Khuong TM, Habets RL, Slabbaert JR, Verstreken P (2010) WASP is activated by phosphatidylinositol-4,5-bisphosphate to restrict synapse growth in a pathway parallel to bone morphogenetic protein signaling. Proc Natl Acad Sci U S A 107:17379-17384. CrossRef Medline

Kidokoro Y, Kuromi H, Delgado R, Maureira C, Oliva C, Labarca P (2004) Synaptic vesicle pools and plasticity of synaptic transmission at the Drosophila synapse. Brain Res Brain Res Rev 47:18-32. CrossRef Medline

Kim SM, Kumar V, Lin YQ, Karunanithi S, Ramaswami M (2009) Fos and Jun potentiate individual release sites and mobilize the reserve synaptic vesicle pool at the Drosophila larval motor synapse. Proc Natl Acad Sci U S A 106:4000-4005. CrossRef Medline

Kim WT, Chang S, Daniell L, Cremona O, Di Paolo G, De Camilli P (2002) Delayed reentry of recycling vesicles into the fusion-competent synaptic vesicle pool in synaptojanin 1 knockout mice. Proc Natl Acad Sci U S A 99:17143-17148. CrossRef Medline

Koenig JH, Ikeda K (1996) Synaptic vesicles have two distinct recycling pathways. J Cell Biol 135:797-808. CrossRef Medline

Krebs CE, Karkheiran S, Powell JC, Cao M, Makarov V, Darvish H, Di Paolo G, Walker RH, Shahidi GA, Buxbaum JD, De Camilli P, Yue Z, PaisánRuiz C (2013) The Sacl domain of SYNJ1 identified mutated in a family with early-onset progressive Parkinsonism with generalized seizures. Hum Mutat 34:1200-1207. CrossRef Medline

Kuromi H, Kidokoro Y (1998) Two distinct pools of synaptic vesicles in single presynaptic boutons in a temperature-sensitive Drosophila mutant, shibire. Neuron 20:917-925. CrossRef Medline

Kuromi H, Kidokoro Y (2000) Tetanic stimulation recruits vesicles from reserve pool via a cAMP-mediated process in Drosophila synapses. Neuron 27:133-143. CrossRef Medline

Kuromi H, Kidokoro Y (2002) Selective replenishment of two vesicle pools depends on the source of $\mathrm{Ca}^{2+}$ at the Drosophila synapse. Neuron 35:333343. CrossRef Medline

Kuromi H, Kidokoro Y (2005) Exocytosis and endocytosis of synaptic vesicles and functional roles of vesicle pools: lessons from the Drosophila neuromuscular junction. Neuroscientist 11:138-147. CrossRef Medline

Lee SY, Wenk MR, Kim Y, Nairn AC, De Camilli P (2004) Regulation of synaptojanin 1 by cyclin-dependent kinase 5 at synapses. Proc Natl Acad Sci U S A 101:546-551. CrossRef Medline

Mani M, Lee SY, Lucast L, Cremona O, Di Paolo G, De Camilli P, Ryan TA (2007) The dual phosphatase activity of synaptojanin 1 is required for both efficient synaptic vesicle endocytosis and reavailability at nerve terminals. Neuron 56:1004-1018. CrossRef Medline

McPherson PS, Garcia EP, Slepnev VI, David C, Zhang X, Grabs D, Sossin WS, Bauerfeind R, Nemoto Y, De Camilli P (1996) A presynaptic inositol-5-phosphatase. Nature 379:353-357. CrossRef Medline

Mochida S, Kobayashi H, Matsuda Y, Yuda Y, Muramoto K, Nonomura Y (1994) Myosin II is involved in transmitter release at synapses formed between rat sympathetic neurons in culture. Neuron 13:1131-1142. CrossRef Medline

Montell C, Jones K, Hafen E, Rubin G (1985) Rescue of the Drosophila phototransduction mutation trp by germline transformation. Science 230:1040-1043. CrossRef Medline

Ori-McKenney KM, McKenney RJ, Huang HH, Li T, Meltzer S, Jan LY, Vale RD, Wiita AP, Jan YN (2016) Phosphorylation of beta-tubulin by the Down syndrome kinase, minibrain/DYRKla, regulates microtubule dynamics and dendrite morphogenesis. Neuron 90:551-563. CrossRef Medline

O’Roak BJ, Vives L, Fu W, Egertson JD, Stanaway IB, Phelps IG, Carvill G, Kumar A, Lee C, Ankenman K, Munson J, Hiatt JB, Turner EH, Levy R, O’Day DR, Krumm N, Coe BP, Martin BK, Borenstein E, Nickerson DA, et al. (2012) Multiplex targeted sequencing identifies recurrently mutated genes in autism spectrum disorders. Science 338:1619-1622. CrossRef Medline

Pauli A, Althoff F, Oliveira RA, Heidmann S, Schuldiner O, Lehner CF, Dickson BJ, Nasmyth K (2008) Cell-type-specific TEV protease cleavage reveals cohesin functions in Drosophila neurons. Dev Cell 14:239-251. CrossRef Medline

Richards DA, Guatimosim C, Betz WJ (2000) Two endocytic recycling routes selectively fill two vesicle pools in frog motor nerve terminals. Neuron 27:551-559. CrossRef Medline

Ringstad N, Nemoto Y, De Camilli P (1997) The SH3p4/Sh3p8/SH3p13 protein family: binding partners for synaptojanin and dynamin via a Grb2-like Src homology 3 domain. Proc Natl Acad Sci U S A 94:8569_ 8574. CrossRef Medline

Rizzoli SO, Betz WJ (2005) Synaptic vesicle pools. Nat Rev Neurosci 6: 57-69. CrossRef Medline

Roseth S, Fykse EM, Fonnum F (1995) Uptake of L-glutamate into rat brain synaptic vesicles: effect of inhibitors that bind specifically to the glutamate transporter. J Neurochem 65:96-103. CrossRef Medline

Ryan TA (1999) Inhibitors of myosin light chain kinase block synaptic vesicle pool mobilization during action potential firing. J Neurosci 19: 1317-1323. Medline

Ryan TA, Smith SJ (1995) Vesicle pool mobilization during action potential firing at hippocampal synapses. Neuron 14:983-989. CrossRef Medline

Saheki Y, De Camilli P (2012) Synaptic vesicle endocytosis. Cold Spring Harb Perspect Biol 4:a005645. CrossRef Medline

Schikorski T, Stevens CF (2001) Morphological correlates of functionally defined synaptic vesicle populations. Nat Neurosci 4:391-395. CrossRef Medline

Schuske KR, Richmond JE, Matthies DS, Davis WS, Runz S, Rube DA, van der Bliek AM, Jorgensen EM (2003) Endophilin is required for synaptic vesicle endocytosis by localizing synaptojanin. Neuron 40:749-762. CrossRef Medline

Soykan T, Maritzen T, Haucke V (2016) Modes and mechanisms of synaptic vesicle recycling. Curr Opin Neurobiol 39:17-23. CrossRef Medline

Tejedor F, Zhu XR, Kaltenbach E, Ackermann A, Baumann A, Canal I, Heisenberg M, Fischbach KF, Pongs O (1995) minibrain: a new protein kinase family involved in postembryonic neurogenesis in Drosophila. Neuron 14:287-301. CrossRef Medline

Verstreken P, Koh TW, Schulze KL, Zhai RG, Hiesinger PR, Zhou Y, Mehta SQ, Cao Y, Roos J, Bellen HJ (2003) Synaptojanin is recruited by endophilin to promote synaptic vesicle uncoating. Neuron 40:733-748. CrossRef Medline

Verstreken P, Ly CV, Venken KJ, Koh TW, Zhou Y, Bellen HJ (2005) Synaptic mitochondria are critical for mobilization of reserve pool vesicles at Drosophila neuromuscular junctions. Neuron 47:365-378. CrossRef Medline

Verstreken P, Ohyama T, Bellen HJ (2008) FM 1-43 labeling of synaptic vesicle pools at the Drosophila neuromuscular junction. Methods Mol Biol 440:349-369. CrossRef Medline

Wu Y, O’Toole ET, Girard M, Ritter B, Messa M, Liu X, McPherson PS, Ferguson SM, De Camilli P (2014) A dynamin 1-, dynamin 3- and clathrin-independent pathway of synaptic vesicle recycling mediated by bulk endocytosis. eLife 3:e01621. CrossRef Medline 